\title{
A new sample of giant radio galaxies from the WENSS survey
}

\section{A multi-frequency radio study of a complete sample: Properties of the radio lobes and their environment}

\author{
A.P. Schoenmakers ${ }^{1,2,5, \star}$, K.-H. Mack ${ }^{3,4}$, A.G. de Bruyn ${ }^{5,6}$, H.J.A. Röttgering ${ }^{2}$, U. Klein ${ }^{4}$, and H. van der Laan ${ }^{1}$ \\ 1 Astronomical Institute, Utrecht University, P.O. Box 80000 , 3508 TA Utrecht, The Netherlands \\ 2 Sterrewacht Leiden, Leiden University, P.O. Box 9513, 2300 RA Leiden, The Netherlands \\ 3 Istituto di Radioastronomia del CNR, Via P. Gobetti 101, I-40129 Bologna, Italy \\ 4 Radioastronomisches Institut der Universität Bonn, Auf dem Hügel 71, D-53121 Bonn, Germany \\ 5 NFRA, P.O. Box 2, 7990 AA Dwingeloo, The Netherlands \\ ${ }^{6}$ Kapteyn Astronomical Institute, University of Groningen, P.O. Box 800, 9700 AV Groningen, The Netherlands
}

Received December 15, 1999; accepted August 2, 2000

\begin{abstract}
We have formed a complete sample of 26 low redshift $(z \lesssim 0.3)$ giant radio galaxies (GRGs) from the WENSS survey, selected at flux densities above $1 \mathrm{Jy}$ at $325 \mathrm{MHz}$. We present $10.5-\mathrm{GHz}$ observations with the $100-\mathrm{m}$ Effelsberg telescope of 18 sources in this sample. These observations, together with similar data of the remaining eight sources, are combined with data from the WENSS, NVSS and GB6 surveys to study the radio properties of the lobes of these sources at arcminute resolution. We investigate radio source asymmetries, equipartition energy densities in the lobes, the presence of lobe pressure evolution with redshift, the spectral age and the density of the environments of these sources. We find that the armlength asymmetries of GRGs are slightly larger than those of smaller sized 3CR radio galaxies and that these are difficult to explain as arising from orientation effects only. We also find indications that the lobes of the GRGs, despite their large sizes, are still overpressured with respect to their environment. Further, we argue that any evolution of lobe pressure with redshift in these large sources (e.g. Cotter 1998) is due to selection effects. For sources which could be used in a spectral ageing analysis, we find spectral ages which are large, typically a few times $10^{7} \mathrm{yr}$. This is comparable to earlier studies of some giant sources and indicates that such large spectral ages are common for this class of radio source. The advance velocities of the radio lobes are typically a few percent of the speed of light, which is higher than those found for smaller, low power $\left(<10^{26.5} \mathrm{~W} \mathrm{~Hz}^{-1}\right.$ at $\left.178 \mathrm{MHz}\right)$ radio sources, and more
\end{abstract}

Send offprint requests to: A.P. Schoenmakers,

e-mail: schoenmakers@astron.nl

* Present address: NFRA, P.O. Box 2, 7990 AA Dwingeloo, The Netherlands. comparable to higher power radio sources. This suggests that the GRGs in our sample are the oldest members of the group of relatively high power radio sources whose radio powers have evolved to their currently observed lower values (cf. Kaiser et al. 1997).

Key words: galaxies: active - intergalactic medium galaxies: jets — radio continuum: galaxies

\section{Introduction}

Giant radio galaxies (GRGs) are the largest radio sources in the Universe which are associated with active galactic nuclei (AGN). A common definition for GRGs is that they are radio sources with a linear size above $12 \mathrm{Mpc}^{1}$. These enormous sizes make them interesting objects to study. Why are they so large? Is it because they grow much faster than other radio galaxies, or are they extremely old radio sources? Which are the properties of their progenitors? Also, because their size is so extreme, they allow us to study their radio structures in detail and to use them as probes of the gaseous environment of their host galaxies on scales of a few hundred kpc to a few Mpc.

Since their discovery in the early seventies, several individual GRGs have been the subject of detailed radio studies at a variety of wavelengths and resolutions (e.g. 3C 236 by Strom \& Willis 1980 and Barthel et al. 1985;

\footnotetext{
${ }^{1}$ We use $H_{0}=50 \mathrm{~km} \mathrm{~s}^{-1} \mathrm{Mpc}^{-1}$ and $q_{0}=0.5$ throughout this paper.
} 
NGC 315 by Willis et al. 1981, NGC 6251 by Perley et al. 1984). However, systematic studies of GRGs as a population have always been hampered by the small number of sources available and by non-uniform selection effects. Since GRGs are large and their radio emission is not very powerful their surface brightness is relatively low. This makes them difficult objects to detect or recognize in most large-scale radio surveys. As a result, a large fraction of the known GRGs have been discovered serendipitously (e.g. Hine 1979; de Bruyn 1989), hence the difficulty in obtaining a uniformly selected sample. The most uniform dataset on GRGs available yet is that of $10.5-\mathrm{GHz}$ observations with the 100-m Effelsberg telescope (Klein et al. 1994; Saripalli et al. 1996; Mack et al. 1997). All sources have been observed in the same way and at similar sensitivities (RMS-noise $\sim 1 \mathrm{mJy}_{\text {beam }}{ }^{-1}$ ), so that the results can be easily compared to each other.

In Paper I (Schoenmakers et al. 1900a; see also Schoenmakers 1999) we present a new sample of 47 GRGs selected from the $325-\mathrm{MHz}$ WENSS survey (Rengelink et al. 1997). In this paper, we will define a complete subsample of 26 sources with a $325-\mathrm{MHz}$ flux density above $1 \mathrm{Jy}$. This is the largest complete sample of GRGs, with well understood selection effects (see Paper I), yet compiled. We have used this sample for several follow-up studies, among which a study of their radio properties using multi-frequency radio data. For this purpose, we have obtained new $10.5-\mathrm{GHz}$ radio data of 18 of these sources using observations with the 100-m Effelsberg telescope; the 8 remaining sources have already been observed with this instrument (Klein et al. 1994; Saripalli et al. 1996; Mack et al. 1997). We have used these data to investigate the high-frequency radio morphology, the magnetic field configuration and, combined with data obtained at lower frequencies, the spectral index distribution and spectral ages (e.g. Mack et al. 1998). The analysis and results of this study are presented here. Subsequent papers will deal with the optical properties of the AGN and their relation with the radio structure, and with the evolution of GRGs, both in terms of cosmological evolution as in terms of intrinsic radio source evolution.

In Sect. 2 we present the complete sample of GRGs and discuss some of its characteristics. Section 3 presents the new $10.5-\mathrm{GHz}$ radio data and lower frequency data for the sources in the sample. In Sect. 4 we derive several source asymmetry parameters and investigate the presence of correlations between these. The ages and lobe advance velocities of several GRGs are derived in Sect. 5, and the energy densities and lobe pressures are derived in Sect. 6. In Sect. 7 we discuss the results, focusing on the spectral ages and the environment of the GRGs. Our conclusions are presented in Sect. 8.

Throughout this paper, a spectral index $\alpha$ is defined according to the relation $S_{\nu} \propto \nu^{\alpha}$ between flux density $S$ and frequency $\nu$.

\section{A complete sample of GRGs}

The GRGs presented in Paper I have been selected from the WENSS survey using the criteria that they should have an angular size above $5^{\prime}$ and a distance from the galactic plane $\geq 12.5$. We find in Paper I that a WENSS radio source is most likely included in the sample if $S_{\text {int }} / \theta_{\max } \gtrsim 0.025 \mathrm{Jy} /$ arcmin, where $S_{\text {int }}$ is the integrated $325-\mathrm{MHz}$ flux density, and $\theta_{\max }$ the largest angular size of the radio source. We have called this the sensitivity limit of our selection.

The total number of sources in the sample of Paper I is 47 , but at low flux density levels (i.e. $\lesssim 200 \mathrm{mJy}$ ) several candidate sources have not yet been identified, and some sources may have been missed because they have not been recognized as single structures. Therefore, we have selected a subsample of 26 sources on the basis of a flux density at $325 \mathrm{MHz}, S_{325}>1 \mathrm{Jy}$. At such high flux densities, it is unlikely that a source has escaped detection or recognition as a GRG.

On basis of our sensitivity limit (see above), it is unlikely that a 1-Jy GRG will be recognized at a redshift below 0.014 , due to its very low surface brightness in that case. However, the lowest redshift source in our sample is NGC 315 at $z=0.0167$, so no source needed to be excluded on basis of this limit.

We have omitted two giant FRI-type (Fanaroff \& Riley 1974) radio sources from the sample: 3C 31 (e.g. Strom et al. 1983) and HB 13 (e.g. Masson 1979). The considerations for doing so were the following. First, the observed size of sources of this class depends strongly on the surface brightness sensitivity of the radio observations; only for edge-brightened FRII-type sources, the angular size and source structure is reasonably well defined because of the presence of hotspots and the usually better outlined radio lobe morphology. The edge-darkened nature of FRI's makes it unlikely that the 1-Jy sample is complete for FRI-type sources. Second, the properties of the radio lobes of FRI-type sources are known to be different. This is shown, for instance, by the different spectral index distribution in the radio lobes (e.g. Jägers 1986; Parma et al. 1999), which may indicate different mechanisms for the acceleration and ageing of the radiating particles.

There are a few remaining sources which are strictly of type FRI, but also show properties commonly found in FRII-type sources, such as hotspots or sharply bound lobe structures. Well known examples of such sources are DA 240, NGC 315 and NGC 6251. Since the measured size of these sources is better constrained, we have left these sources in the sample, although they are excluded from many of the analyses in this paper. They are indicated as type "FRI/II" in Table 1.

Our 1-Jy sample therefore contains all non FRI-type sources with a $325-\mathrm{MHz}$ flux density above $1 \mathrm{Jy}$, an angular size above $5^{\prime}$, a linear size above $1 \mathrm{Mpc}$, a declination above $+28^{\circ}$, a distance from the galactic plane $>12.5^{\circ}$ 
Table 1. List of the 26 sources which form the "1-Jy sample" of GRGs. Column 1 gives the name of the source in IAU-notation, with coordinates in B 1950.0; Col. 2 gives an alternative, more common name, if available; Col. 3 indicates whether it is one of the newly discovered GRGs ("N") or one of the formerly known, or "old" GRGs ("O"); Col. 4 gives the redshift of the source; Col. 5 gives the radio morphological type. A "B" indicates it is a broad-line radio galaxy, i.e. the Hydrogen Balmer lines have broad components; a "Q" indicates a quasar-like spectrum (broad lines, blue continuum). Column 6 gives the projected linear size in Mpc. For references concerning the properties of the old GRGs we refer to Paper I

\begin{tabular}{|c|c|c|c|c|c|}
\hline $\begin{array}{c}(1) \\
\text { IAU name }\end{array}$ & $\begin{array}{c}(2) \\
\text { Alt. name }\end{array}$ & (3) & $\begin{array}{c}(4) \\
z\end{array}$ & $\begin{array}{l}(5) \\
\text { Type } \\
\text { FR } \\
\end{array}$ & $\begin{array}{c}(6) \\
D \\
{[\mathrm{Mpc}]}\end{array}$ \\
\hline В $0050+402$ & & $\mathrm{O}$ & 0.1488 & II & 1.5 \\
\hline В $0055+300$ & NGC 315 & $\mathrm{O}$ & 0.0167 & $\mathrm{I} / \mathrm{II}$ & 1.7 \\
\hline В $0109+492$ & $3 \mathrm{C} 35$ & $\mathrm{O}$ & 0.0670 & II & 1.1 \\
\hline В $0136+396$ & 4C 39.04 & $\mathrm{O}$ & 0.2107 & II & 1.6 \\
\hline B $0157+405$ & $4 \mathrm{C} 40.09$ & $\mathrm{O}$ & 0.0827 & $\mathrm{I} / \mathrm{II}$ & 1.9 \\
\hline В $0211+326$ & & $\mathrm{~N}$ & 0.2605 & II & 1.6 \\
\hline В $0309+411$ & & $\mathrm{O}$ & 0.1340 & II-B & 1.8 \\
\hline В $0648+733$ & & $\mathrm{~N}$ & 0.1145 & II & 1.9 \\
\hline В $0658+490$ & & $\mathrm{~N}$ & 0.0650 & $\mathrm{I} / \mathrm{II}$ & 1.9 \\
\hline B $0745+560$ & DA 240 & $\mathrm{O}$ & 0.0356 & $\mathrm{I} / \mathrm{II}$ & 2.0 \\
\hline В $0813+758$ & & $\mathrm{~N}$ & 0.2324 & II-B & 2.3 \\
\hline В $0945+734$ & 4C 73.08 & $\mathrm{O}$ & 0.0581 & II & 1.5 \\
\hline B $1003+351$ & $3 \mathrm{C} 236$ & $\mathrm{O}$ & 0.0989 & II & 5.7 \\
\hline B $1209+745$ & 4CT 74.17 & $\mathrm{O}$ & 0.107 & II & 1.2 \\
\hline B $1213+422$ & & $\mathrm{~N}$ & 0.2426 & II-B & 1.6 \\
\hline B $1309+412$ & & $\mathrm{O}$ & 0.1103 & II & 1.0 \\
\hline B $1312+698$ & DA 340 & $\mathrm{O}$ & 0.106 & II & 1.3 \\
\hline B $1358+305$ & & $\mathrm{O}$ & 0.206 & II & 2.6 \\
\hline B $1426+295$ & & $\mathrm{~N}$ & 0.0870 & II & 1.9 \\
\hline B $1450+333$ & & $\mathrm{~N}$ & 0.249 & II & 1.7 \\
\hline B $1543+845$ & & $\mathrm{~N}$ & 0.201 & II & 2.1 \\
\hline B $1626+518$ & & $\mathrm{O}$ & 0.0547 & II & 1.6 \\
\hline B $1637+826$ & NGC 6251 & $\mathrm{O}$ & 0.023 & $\mathrm{I} / \mathrm{II}$ & 3.0 \\
\hline B $1918+516$ & & $\mathrm{~N}$ & $0.284^{a}$ & II & 2.3 \\
\hline B $2043+749$ & $4 \mathrm{C} 74.26$ & $\mathrm{O}$ & 0.104 & II-Q & 1.6 \\
\hline B $2147+816$ & & $\mathrm{~N}$ & 0.1457 & II & 3.7 \\
\hline
\end{tabular}

Notes:

$a$-Redshift still uncertain (see text and Paper I).

\section{Radio data and radio spectra of the 1-Jy sample}

In this section we present new $10.5-\mathrm{GHz}$ radio data of 18 of the 26 sources in the 1-Jy sample of GRGs. We have measured total and lobe flux densities of all sources at several frequencies between $325 \mathrm{MHz}$ and $10.5 \mathrm{GHz}$. Further, we present the radio spectra of the sources and their radio lobes.

\subsection{The 10.5-GHz observations}

Eight sources in our sample were already observed at 10.5 GHz with the 100-m Effelsberg telescope (Klein et al. 1994; Saripalli et al. 1996; Mack et al. 1997). We have observed the 18 remaining sources with the same instrument and achieving a similar sensitivity of $\sigma_{\text {rms }} \approx 1 \mathrm{mJy}$ beam $^{-1}$.

The observations have been carried out in multiple observing sessions between December 1995 and April 1998, using the 4-horn receiver operating at a central frequency of $10.45 \mathrm{GHz}$ and employing a bandwidth of $300 \mathrm{MHz}$. The beam size at this frequency is $69^{\prime \prime}$ (FWHM). For a detailed description of the basic observational technique and data reduction procedure we refer to the paper by Gregorini et al. (1992). The calibration of the flux density scale has been achieved by mapping 3C 286 and 3C 295, with the flux density scale adopted from Baars et al. (1977). Field sizes and map centers are compiled in Table 2. Mapping was performed by scanning the telescope in azimuth with a speed of $40^{\prime} / \mathrm{min}$, with a scan separation of $20^{\prime \prime}$ in elevation and using all four horns. Difference maps have been computed from all horns to efficiently suppress atmospheric disturbances of the signal. Following the usual restoration technique of the differential maps (Emerson et al. 1979) the maps were transformed into a right ascension - declination system. All maps, with the exception of B $0309+411$, have been CLEANed to remove sidelobes, applying the algorithm described by Klein \& Mack (1995). During the observations of B $0309+411$, snow was collected on the dish of the telescope. Since this has affected the beam-shape, cleaning was not performed on this source. Also, the snow led to problems with calibrating the data. Therefore the flux density of B $0309+411$ is estimated to be only correct to $20 \%$. The individual coverages (Table 2) of each source have been averaged to give the final Stokes' $I, Q$ and $U$ maps. Contour plots of the radio maps have been presented in Appendix A.

\subsection{Other radio data}

\subsection{1. $325-\mathrm{MHz}$ data}

We have measured the $325-\mathrm{MHz}$ flux densities and morphological parameters on the radio maps of the WENSS survey. The mosaicing technique used in the observations difficulty we had in actually identifying it; an independent confirmation is needed. 
Table 2. Observing log of the 10.5-GHz observations. Column 1 gives the name of the source. Columns 2 and 3 give the B1950.0 coordinates of the center of the map. Column 4 gives the number of coverages used in the final maps (i.e. after removing those which suffered from bad atmospheric conditions). The number of coverages used in the $I, Q$ and $U$ maps are the same. Column 5 gives the size of the area covered. Column 6 gives the observing dates and the number of coverages on that date in the format "number of coverages:month/year". Columns 7 and 8 give the RMS-noise in the total power maps and in the $Q, U$ maps

\begin{tabular}{|c|c|c|c|c|c|c|c|}
\hline (1) & (2) & (3) & $(4)$ & (5) & (6) & $(7)$ & $(8)$ \\
\hline Source & $\begin{array}{c}\mathrm{RA} \\
{[\mathrm{h} \mathrm{m} \mathrm{s}]}\end{array}$ & $\begin{array}{l}\text { Decl. } \\
{\left[\begin{array}{lll}0 & \prime & 11\end{array}\right]}\end{array}$ & Cov. & $\begin{array}{c}\text { Mapsize } \\
{\left[\begin{array}{l}\prime \\
\end{array}{ }^{\prime}\right]}\end{array}$ & Obs. dates & $\begin{array}{l}\sigma_{I} \\
{[\mathrm{mJy}}\end{array}$ & $\begin{array}{c}\sigma_{Q, U} \\
\mathrm{eam}^{-1}\end{array}$ \\
\hline B $0109+492$ & 010907.0 & 491300.0 & 10 & $38 \times 18$ & $11: 12 / 95$ & 1.1 & 0.3 \\
\hline B $0157+405$ & 015727.8 & 403423.0 & 11 & $41 \times 20$ & $1: 05 / 94 ; 3: 08 / 94 ; 2: 11 / 94 ; 1: 12 / 94 ; 4: 12 / 95$ & 1.3 & 0.3 \\
\hline B $0211+326$ & 021119.5 & 323718.0 & 14 & $31 \times 10$ & $14: 12 / 96$ & 0.9 & 0.3 \\
\hline В $0309+411$ & 030949.9 & 410833.0 & 13 & $34 \times 13$ & $7: 12 / 96 ; 8: 01 / 97$ & 1.1 & 0.3 \\
\hline В $0648+734$ & 064814.0 & 732420.5 & 10 & $39 \times 18$ & $10: 12 / 95$ & 1.0 & 0.3 \\
\hline B $0658+490$ & 065834.4 & 490133.0 & 11 & $45 \times 24$ & $4: 12 / 95 ; 12: 12 / 96 ; 1: 01 / 97$ & 1.1 & 0.3 \\
\hline B $0813+758$ & 081335.3 & 754853.0 & 9 & $35 \times 14$ & $10: 12 / 96$ & 1.0 & 0.3 \\
\hline B $1209+745$ & 120932.0 & 743634.1 & 9 & $34 \times 13$ & $9: 12 / 96$ & 1.0 & 0.3 \\
\hline B $1213+422$ & 121338.8 & 421617.0 & 7 & $31 \times 10$ & $7: 12 / 96$ & 1.3 & 0.4 \\
\hline B $1312+698$ & 131225.7 & 695255.0 & 9 & $32 \times 11$ & $9: 12 / 96$ & 0.9 & 0.3 \\
\hline B $1426+295$ & 142609.0 & 293053.0 & 12 & $43 \times 22$ & $13: 12 / 96$ & 1.1 & 0.3 \\
\hline B $1450+333$ & 145058.4 & 332052.0 & 10 & $32 \times 11$ & $10: 04 / 98$ & 0.6 & 0.3 \\
\hline B $1543+845$ & 154354.9 & 843225.0 & 11 & $33 \times 12$ & $14: 04 / 98$ & 0.6 & 0.3 \\
\hline B $1626+518$ & 162700.0 & 515351.0 & 12 & $46 \times 25$ & $12: 12 / 95$ & 1.3 & 0.3 \\
\hline B $1918+516$ & 191809.6 & 513730.0 & 7 & $33 \times 12$ & $4: 12 / 96 ; 3: 01 / 97$ & 1.3 & 0.4 \\
\hline B $2147+816$ & 214648.2 & 814011.0 & 11 & $45 \times 24$ & $5: 12 / 96 ; 5: 04 / 98 ; 7: 08 / 98$ & 0.9 & 0.3 \\
\hline
\end{tabular}

for WENSS (see Rengelink et al. 1997 for a description) results in a highly uniform coverage of the $(u, v)$-plane, with baselines as short as $\sim 40 \lambda$. WENSS is therefore potentially well suited to obtain accurate flux densities of extended structures. However, since the $(u, v)$-plane is not as well sampled as with continuous observations, in case of complicated and very extended source structures WENSS cannot map all source components reliably. This is particularly notable in sources such as DA 240 or NGC 6251. To illustrate this problem we present in Fig. 1 a map of the source DA 240 as it appears in the WENSS and as it is published by Mack et al. (1997) from a complete 12-hr WSRT synthesis observation. In the latter case, not only the noise level is much lower, but also the fainter extended radio structures are much better reproduced. Since Mack et al. have published maps resulting from full synthesis WSRT observations for the four spatially largest objects in our sample (NGC 315, DA 240, 3C 236, NGC 6251), which will suffer most from this effect, we have measured the flux densities using their maps.

\subsubsection{4-GHz data}

All sources have also been observed at $1.4 \mathrm{GHz}$ in the NVSS survey (Condon et al. 1998). The observations for the NVSS survey were done in a "snap-shot" mode, using the VLA in its D-configuration with baselines down to $\sim 170 \lambda$ only. Both these aspects seriously degrade the sensitivity for structures above $10^{\prime}-15^{\prime}$ in angular size. We therefore only present $1.4-\mathrm{GHz}$ flux densities from the NVSS survey for sources smaller than $10^{\prime}$.

\subsection{3. $4.85-\mathrm{GHz}$ data}

Five GRGs in our sample (NGC 315, DA 240, 3C 236, NGC 6251 and B 1358+295) have been observed at $4.75 \mathrm{GHz}$ with the 100-m Effelsberg telescope (Parma et al. 1996; Mack et al. 1997). The beam size for these observations is $\sim 2 ! 5$ (FWHM). For sources without $5-\mathrm{GHz}$ Effelsberg data we have used the $4.85-\mathrm{GHz}$ Greenbank survey (GB6; Gregory et al. 1996) to measure flux densities. This survey has mapped the northern sky up to a declination of $+75^{\circ}$, using a beam size of $3.2 \times 3$ '. (FWHM). For sources above $+75^{\circ}$ declination, we have no $5-\mathrm{GHz}$ flux densities. The flux densities in the source catalogue of the GB6 survey were obtained by fitting Gaussians to the observed sources, which, since all GRGs in our sample are larger than the beam size of the GB6 survey, gives unreliable results in our case. Therefore, we have retrieved the original digital FITS-format images, using the SkyView database, and measured the flux densities directly from the maps. Background offsets in the maps have been determined by measuring the mean flux density in an area directly surrounding 

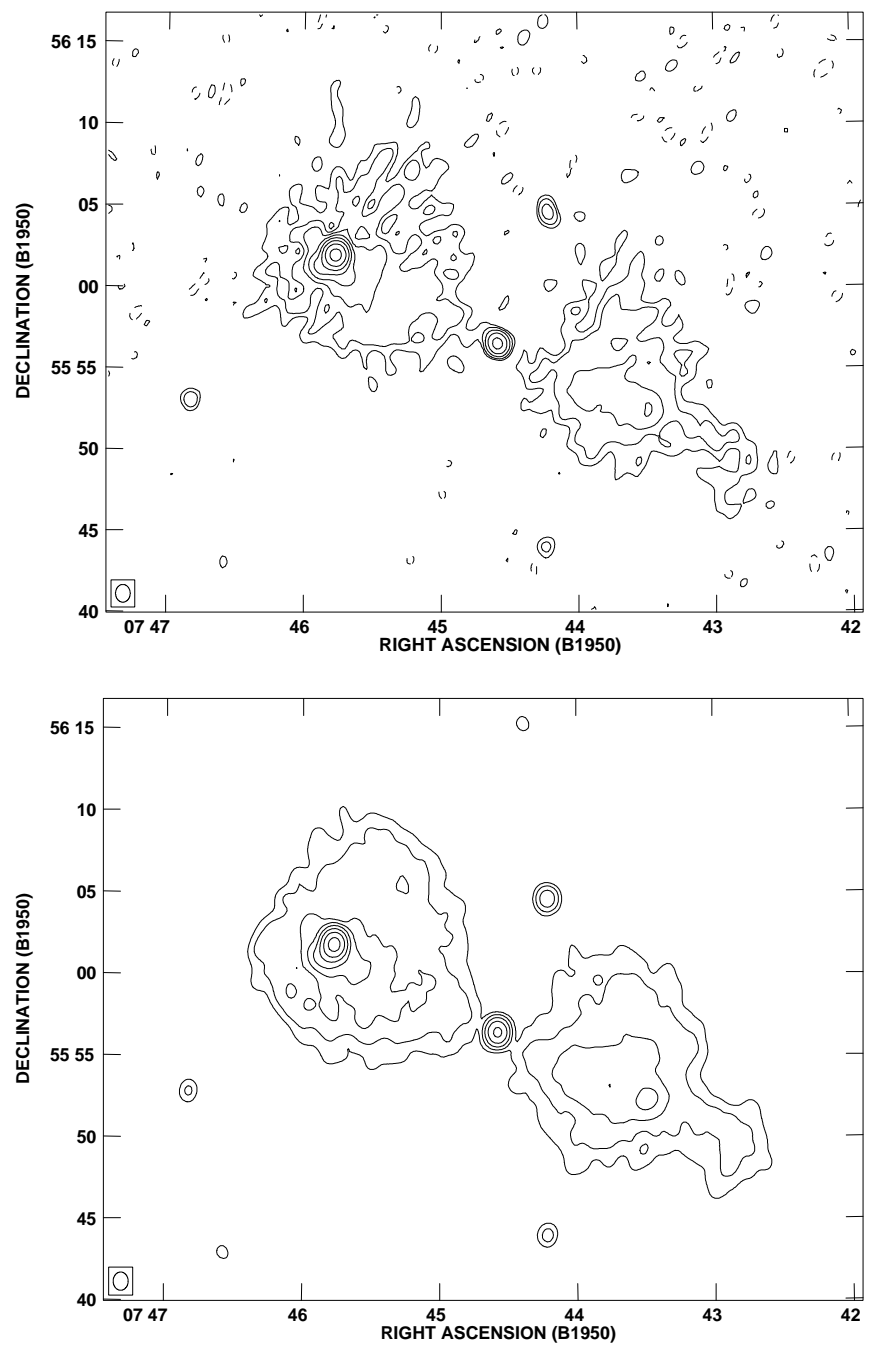

Fig. 1. Two contour plots of the giant radio source DA 240 at a frequency of $325 \mathrm{MHz}$. The upper plot is from the WENSS survey. Contours are drawn at $15 \times(-1,1,2,4,8,16,32,64,128)$ mJy beam ${ }^{-1}$. The lower plot is from a $325-\mathrm{MHz}$ WSRT observation by Mack et al. (1997). Contours are at the same levels as in the top plot

the source, carefully omitting any significant sources in this area. Discrete sources which overlap with the radio structure of the GRGs have been identified in the higher resolution NVSS and $10.5-\mathrm{GHz}$ Effelsberg radio maps. Their contribution to the measured $4.85-\mathrm{GHz}$ flux density has been subtracted by estimating their $4.85-\mathrm{GHz}$ flux density using a power-law interpolation of their 1.4 and 10.5-GHz flux densities.

\subsection{Radio spectra}

We have measured the total integrated flux densities, $S_{\text {int }}$, at $325 \mathrm{MHz}, 4.8 \mathrm{GHz}$ and $10.5 \mathrm{GHz}$. We have also measured the flux densities of the lobes separately at
$325 \mathrm{MHz}, 1.4 \mathrm{MHz}$ (for sources below 10' in angular size), $4.8 \mathrm{GHz}$ (for sources above $10^{\prime}$ in angular size and declination below $+75^{\circ}$ ) and $10.5 \mathrm{GHz}$. Only in the case of B $0309+411$ we have not measured the lobe flux densities at $10.5 \mathrm{GHz}$ due to the strongly dominating radio core at that frequency. The flux densities have been tabulated in Table 3 .

The radio spectra of the sources with more than two flux density measurements are plotted in Fig. 2, based on the flux densities from Table 3. We have used separate signs for the total integrated flux densities and those of the two lobes. Not all sources have been plotted here; similar radio spectra of the sources B $0055+300$ (NGC 315), B 1003+351 (3C 236), B 0745+560 (DA 240) and B 1637+826 (NGC 6251) can be found in Mack et al. (1997); for the source B $1358+305$ we refer to the paper by Parma et al. (1996). The source B $2147+815$ has not been plotted since we only have data at two frequencies for this source (see Table 3).

For several sources the spectrum of the total integrated emission clearly steepens towards higher frequencies (e.g. B $0157+405$, B $0648+733$, B $0945+734$ and B $1312+698$ ). This is usually a sign of spectral ageing of the radiating particles in the source. In other cases the spectrum appears to flatten (e.g. B 0309+411, B 1626+518). Since all these sources have bright radio cores at $10.5 \mathrm{GHz}$, this must be the result of the radio core having a flat, or inverted, spectrum.

\section{Radio source asymmetries}

In this section we measure the asymmetries in armlength, flux density, spectral index, etc. We compare the values and correlations we find for the GRGs with results obtained for samples of smaller-sized sources.

\subsection{Morphological asymmetries}

Asymmetries in the morphology of a radio source are common and may reflect asymmetries in their environment. McCarthy et al. (1991), for instance, find that in a sample of powerful 3CR radio sources there is a correlation between the side of the source with the shortest arm and the side of the source with the highest optical emission-line flux. If a larger intensity of the line emission is due to a higher amount of gas, then the advance of the radio lobe on that side of the source may have been slowed down. On the other hand, the filling factor of this gas may be too low to play an important role in the dynamical evolution of the radio lobe (e.g. Begelmann \& Cioffi 1989). Also, at large distances (i.e. $\gtrsim 100 \mathrm{kpc}$ ) from the host galaxy and the AGN, this warm gas is difficult to detect by emission-line studies even if it were abundant, so that identifying the side with the highest gas density around 

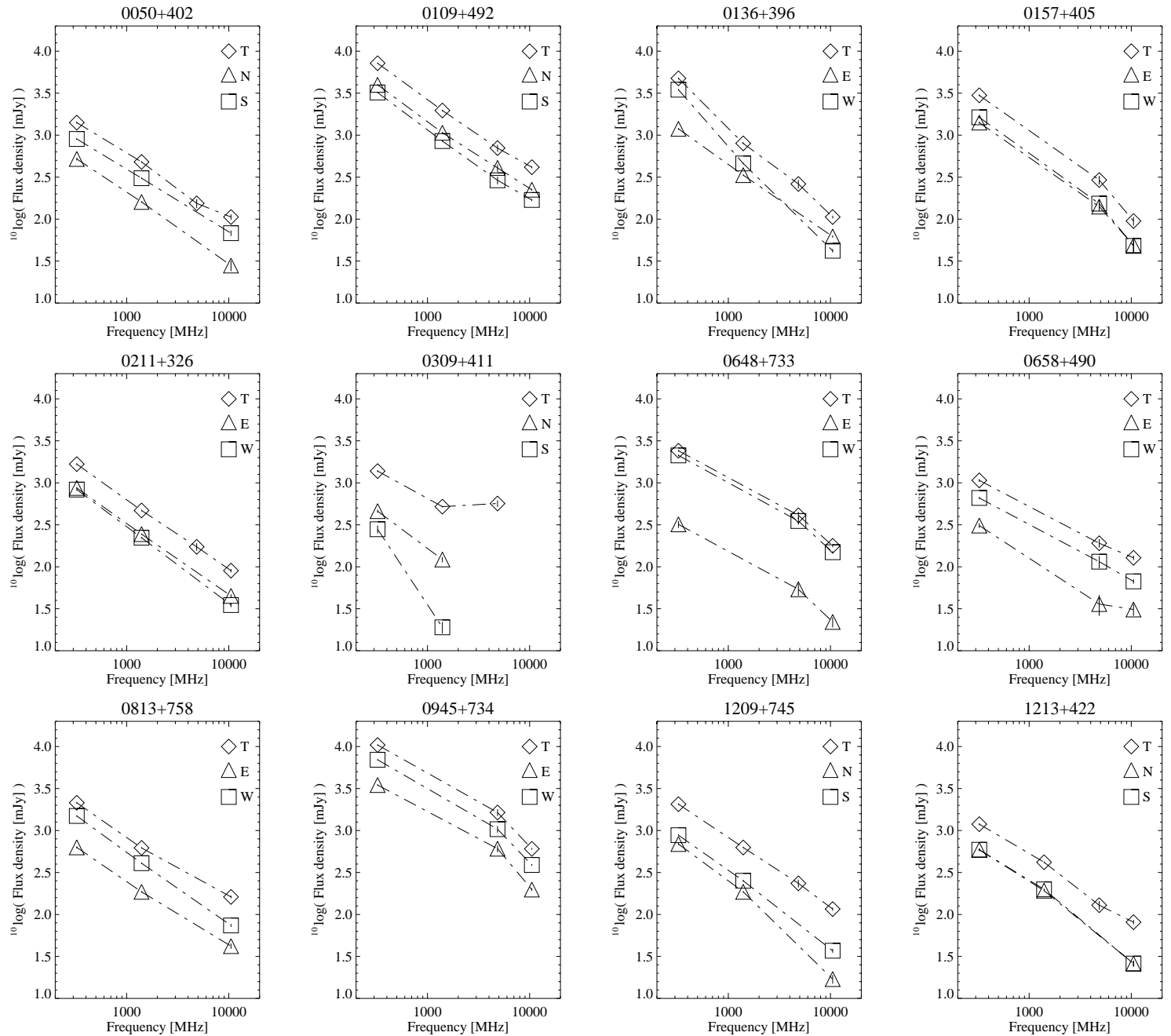

$0945+734$
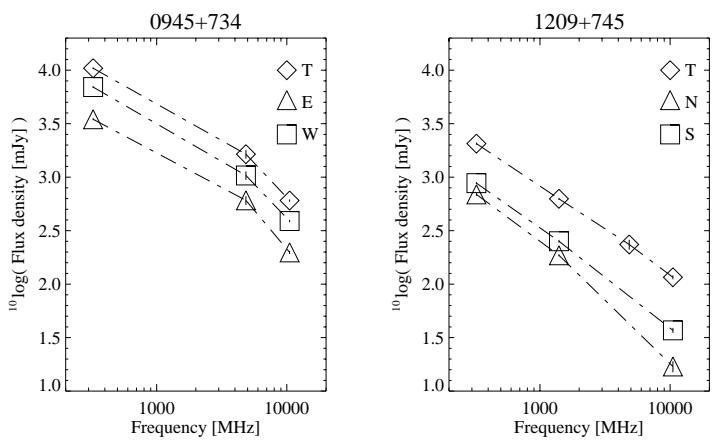

$1213+422$
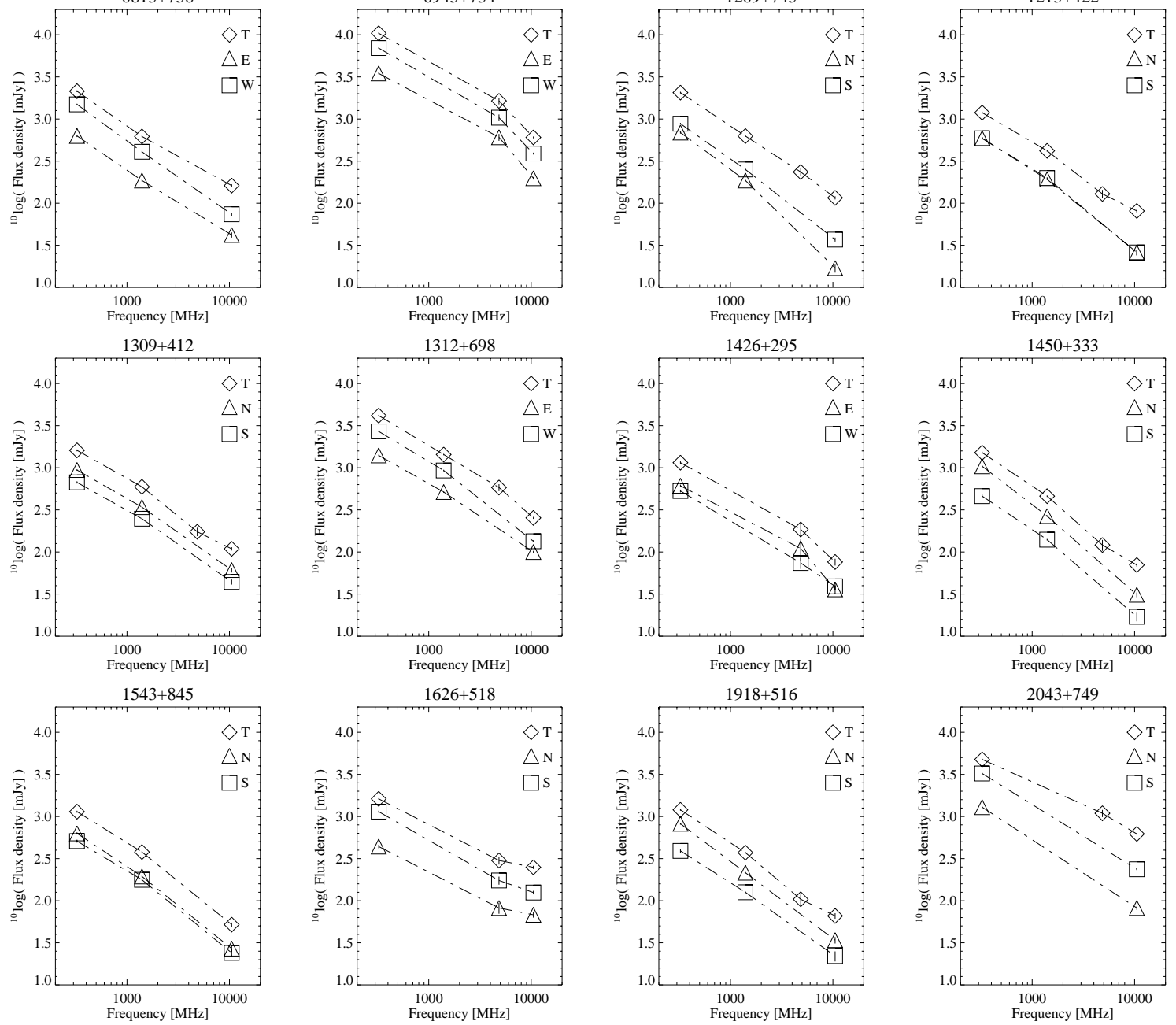

Fig. 2. Radio spectra of the GRGs and their radio lobes. For each source we have plotted the total integrated flux density (indicated by "T"), and the integrated flux density of the two lobes (indicated by "E", "W", etc.) where available. The name of the source is indicated above each panel. See the text for more details 


\begin{tabular}{|c|c|c|c|c|c|c|c|c|c|c|c|c|c|}
\hline (1) & $(2)$ & (3) & $\begin{array}{c}(4) \\
-325 \mathrm{MHz}\end{array}$ & (5) & (6) & $\begin{array}{c}\stackrel{(7)}{ } \\
-1.4 \mathrm{GHz}\end{array}$ & (8) & (9) & $\begin{array}{c}(10) \\
4.8 \mathrm{GHz}\end{array}$ & (11) & (12) & $\begin{array}{c}(13) \\
10.5 \mathrm{GHz}\end{array}$ & (14) \\
\hline Source & $\mathrm{A}, \mathrm{B}$ & $\begin{array}{l}S_{\mathrm{int}} \\
{[\mathrm{Jy}]}\end{array}$ & $\begin{array}{c}S_{A} \\
{[\mathrm{Jy}]}\end{array}$ & $\begin{array}{c}S_{B} \\
{[\mathrm{Jy}]}\end{array}$ & $\begin{array}{c}S_{\mathrm{int}} \\
{[\mathrm{mJy}]}\end{array}$ & $\begin{array}{c}S_{A} \\
{[\mathrm{mJy}]}\end{array}$ & $\begin{array}{c}S_{B} \\
{[\mathrm{mJy}]}\end{array}$ & $\begin{array}{c}S_{\mathrm{int}} \\
{[\mathrm{mJy}]}\end{array}$ & $\begin{array}{c}S_{A} \\
{[\mathrm{mJy}]}\end{array}$ & $\begin{array}{c}S_{B} \\
{[\mathrm{mJy}]}\end{array}$ & $\begin{array}{c}S_{\mathrm{int}} \\
{[\mathrm{mJy}]}\end{array}$ & $\begin{array}{c}S_{A} \\
{[\mathrm{mJy}]}\end{array}$ & $\begin{array}{c}S_{B} \\
{[\mathrm{mJy}]}\end{array}$ \\
\hline В $0050+402$ & $\mathrm{~N}, \mathrm{~S}$ & $1.41 \pm 0.04$ & $0.52 \pm 0.02$ & $90 \pm 0.02$ & 48 & $160 \pm 4$ & $307 \pm 7$ & $154 \pm 19$ & & & $106 \pm 8$ & $28 \pm 4$ & $68 \pm 5$ \\
\hline B $0055+300^{a}$ & $\mathrm{E}, \mathrm{W}$ & $9.71 \pm 0.18$ & $2.53 \pm 0.06$ & $3.55 \pm 0.10$ & & & & $2460 \pm 70$ & $296 \pm 40$ & $569 \pm 50$ & $2056 \pm 50$ & $271 \pm 50$ & $646 \pm 80$ \\
\hline В $0109+492$ & $\mathrm{~N}, \mathrm{~S}$ & $7.19 \pm 0.15$ & $3.98 \pm 0.08$ & $3.22 \pm 0.06$ & $1965 \pm 40$ & $1067 \pm 21$ & $851 \pm 19$ & $702 \pm 67$ & $407 \pm 40$ & $290 \pm 30$ & $415 \pm 11$ & $224 \pm 6$ & $169 \pm 5$ \\
\hline В $0136+396$ & $\mathrm{E}, \mathrm{W}$ & $4.75 \pm 0.10$ & $1.19 \pm 0.03$ & $3.48 \pm 0.07$ & $798 \pm 16$ & $334 \pm 7$ & $460 \pm 10$ & $262 \pm 30$ & & & $106 \pm 4$ & $62 \pm 2$ & $42 \pm 2$ \\
\hline В $0157+405$ & $\mathrm{E}, \mathrm{W}$ & $2.98 \pm 0.08$ & $1.41 \pm 0.04$ & $1.63 \pm 0.04$ & & & & $292 \pm 35$ & $141 \pm 17$ & $153 \pm 18$ & $95 \pm 11$ & $49 \pm 8$ & $48 \pm 8$ \\
\hline В $0211+326$ & $\mathrm{E}, \mathrm{W}$ & $1.67 \pm 0.04$ & $0.87 \pm 0.02$ & $0.83 \pm 0.02$ & $469 \pm 10$ & $245 \pm 5$ & $222 \pm 5$ & $173 \pm 18$ & & & $90 \pm 4$ & $45 \pm 2$ & $35 \pm 2$ \\
\hline В $0309+411^{b}$ & $\mathrm{~N}, \mathrm{~S}$ & $1.38 \pm 0.04$ & $0.46 \pm 0.02$ & $0.28 \pm 0.03$ & $520 \pm 11$ & $122 \pm 9$ & $19 \pm 3$ & $567 \pm 50$ & & $550 \pm 110$ & & & \\
\hline В $0648+733$ & $\mathrm{E}, \mathrm{W}$ & $2.41 \pm 0.06$ & $0.32 \pm 0.03$ & $2.12 \pm 0.05$ & & & & $411 \pm 40$ & $54 \pm 9$ & $351 \pm 33$ & $178 \pm 7$ & $22 \pm 3$ & $149 \pm 4$ \\
\hline В $0658+490^{c}$ & $\mathrm{E}, \mathrm{W}$ & $1.07 \pm 0.04$ & $0.31 \pm 0.02$ & $0.66 \pm 0.02$ & & & & $191 \pm 22$ & $36 \pm 10$ & $115 \pm 19$ & $128 \pm 8$ & $31 \pm 5$ & $67 \pm 4$ \\
\hline В $0745+560^{a}$ & $\mathrm{E}, \mathrm{W}$ & $17.05 \pm 0.35$ & $10.21 \pm 0.21$ & $6.07 \pm 0.13$ & & & & $1780 \pm 50$ & $1187 \pm 23$ & $457 \pm 20$ & $1041 \pm 47$ & $625 \pm 20$ & $165 \pm 15$ \\
\hline В $0813+758$ & $\mathrm{E}, \mathrm{W}$ & $2.14 \pm 0.05$ & $0.63 \pm 0.02$ & $1.49 \pm 0.03$ & $621 \pm 13$ & $186 \pm 5$ & $408 \pm 9$ & & & & $162 \pm 6$ & $42 \pm 3$ & $74 \pm 3$ \\
\hline B $0945+734$ & $\mathrm{E}, \mathrm{W}$ & $10.43 \pm 0.21$ & $3.48 \pm 0.08$ & $6.96 \pm 0.14$ & & & & $1641 \pm 150$ & 607 & $1037 \pm 95$ & $605 \pm 15$ & $198 \pm 7$ & $389 \pm 10$ \\
\hline B $1003+351^{a}$ & $\mathrm{E}, \mathrm{W}$ & $13.13 \pm 0.26$ & $2.18 \pm 0.08$ & $2.99 \pm 0.06$ & & & & $2200 \pm 45$ & $213 \pm 20$ & $401 \pm 20$ & $1208 \pm 28$ & $177 \pm 10$ & $166 \pm 10$ \\
\hline B $1209+745$ & $\mathrm{~N}, \mathrm{~S}$ & $2.06 \pm 0.05$ & $0.69 \pm 0.02$ & $0.88 \pm 0.02$ & $627 \pm 13$ & $186 \pm 4$ & $252 \pm 7$ & $235 \pm 25$ & & & $116 \pm 5$ & $17 \pm 2$ & $37 \pm 2$ \\
\hline B $1213+422$ & $\mathrm{~N}, \mathrm{~S}$ & $9 \pm 0.03$ & $0.59 \pm 0.02$ & $0.59 \pm 0.02$ & $419 \pm 9$ & $192 \pm 4$ & $199 \pm 4$ & $129 \pm 15$ & & & $81 \pm 5$ & $26 \pm 2$ & $26 \pm 2$ \\
\hline В $1309+412$ & $\mathrm{~N}, \mathrm{~S}$ & \pm 0.04 & $0.94 \pm 0.02$ & $0.67 \pm 0.02$ & $594 \pm 12$ & $341 \pm 7$ & $248 \pm 5$ & $174 \pm 20$ & & & $109 \pm 5$ & $61 \pm 4$ & $44 \pm 3$ \\
\hline В $1312+698$ & $\mathrm{E}, \mathrm{W}$ & $4.16 \pm 0.09$ & $1.40 \pm 0.03$ & $2.70 \pm 0.06$ & $1436 \pm 29$ & $515 \pm 11$ & $928 \pm 19$ & $583 \pm 55$ & & & $254 \pm 7$ & $100 \pm 3$ & $134 \pm 4$ \\
\hline B $1358+305^{d}$ & $\mathrm{~N}, \mathrm{~S}$ & $1.84 \pm 0.04$ & $1.32 \pm 0.03$ & $0.52 \pm 0.02$ & $451 \pm 10$ & $318 \pm 7$ & $136 \pm 4$ & $122 \pm \quad 4$ & $95 \pm 4$ & $28 \pm \quad 4$ & $52 \pm 2$ & $34 \pm 2$ & $19 \pm 2$ \\
\hline B $1426+295^{e}$ & $\mathrm{E}, \mathrm{W}$ & $1.15 \pm 0.04$ & $0.61 \pm 0.02$ & $0.53 \pm 0.02$ & & & & $185 \pm 36$ & $110 \pm 31$ & $74 \pm 11$ & $76 \pm 7$ & $36 \pm 4$ & $39 \pm 4$ \\
\hline В $1450+333$ & $\mathrm{~N}, \mathrm{~S}$ & $1.51 \pm 0.04$ & $1.04 \pm 0.03$ & $0.46 \pm 0.02$ & $460 \pm 10$ & $267 \pm 6$ & $140 \pm 3$ & $121 \pm 18$ & & & $70 \pm 3$ & $31 \pm 2$ & $17 \pm 2$ \\
\hline B $1543+845$ & $\mathrm{~N}, \mathrm{~S}$ & $1.14 \pm 0.03$ & $0.63 \pm 0.02$ & $0.51 \pm 0.02$ & $378 \pm 9$ & $194 \pm 4$ & $177 \pm 4$ & & & & $52 \pm 3$ & $27 \pm 2$ & $24 \pm 2$ \\
\hline B $1626+518^{f}$ & $\mathrm{~N}, \mathrm{~S}$ & $1.62 \pm 0.03$ & $0.44 \pm 0.02$ & $1.14 \pm 0.03$ & & & & $300 \pm 31$ & $82 \pm 12$ & $174 \pm 19$ & $249 \pm 11$ & $68 \pm 5$ & $125 \pm 6$ \\
\hline B $1637+826^{g}$ & $\mathrm{E}, \mathrm{W}$ & $11.55 \pm 0.23$ & $2.70 \pm 0.09$ & $4.01 \pm 0.10$ & & & & & & & $1558 \pm 40$ & $85 \pm 20$ & $216 \pm 15$ \\
\hline В $1918+516$ & $\mathrm{~N}, \mathrm{~S}$ & $1.20 \pm 0.03$ & $0.82 \pm 0.02$ & $0.39 \pm 0.02$ & $372 \pm 8$ & $215 \pm 5$ & $126 \pm 3$ & $104 \pm 14$ & & & $66 \pm 5$ & $34 \pm 3$ & $22 \pm 3$ \\
\hline B $2043+749^{h}$ & $\mathrm{~N}, \mathrm{~S}$ & $4.76 \pm 0.10$ & $1.29 \pm 0.03$ & $3.24 \pm 0.07$ & & & & $1091 \pm 105$ & & & $621 \pm 14$ & $82 \pm 4$ & $236 \pm 6$ \\
\hline B $2147+816$ & $\mathrm{~N}, \mathrm{~S}$ & $1.06 \pm 0.05$ & $0.46 \pm 0.02$ & $0.56 \pm 0.02$ & & & & & & & $111 \pm 7$ & $47 \pm 4$ & $52 \pm 4$ \\
\hline
\end{tabular}

Notes: $a-4.75-\mathrm{GHz}$ flux densities from Mack et al. (1997); $b$-Lobe flux densities measured after subtracting unresolved source at core position. No 10.5-GHz lobe flux densities given because of strong core dominance at this frequency. $c-4.85-\mathrm{GHz}$ flux density of eastern lobe after subtracting the estimated core flux density (by interpolation of the 1.4 and 10.5-GHz flux density); $d-4.75-\mathrm{GHz}$ flux densities from Parma et al. (1996); $e-\mathrm{At} 4.85 \mathrm{GHz}$ (GB6), confused with bright source of estimated flux density of $165 \mathrm{mJy}$ (by power-law interpolation of the 1.4 and $10.5 \mathrm{GHz}$ flux density); $f-4.85-\mathrm{GHz}$ flux density of southern lobe after subtracting the estimated core flux density (by power-law interpolation of the 1.4 and 10.5-GHz flux density); $g$-Flux densities of the western lobe exclude the core-jet structure. Also, no 4.8-GHz data available; $h$-No lobe flux densities measured at $4.85 \mathrm{GHz}$ due to the dominating radio core. 
the lobes becomes extremely difficult for larger sources. If line emitting gas or clouds are dynamically unimportant for large sources, then the armlength asymmetries of radio galaxies may reflect asymmetries in the distribution of the hot $\left(\sim 10^{7} \mathrm{~K}\right)$ diffuse IGM around the host galaxy. In principle, GRGs thus allow us to investigate the uniformity of the IGM on scales up to a few Mpc, which is well outside of the reach of current X-ray instruments, apart from a few very luminous clusters.

However, armlength asymmetries can also be a result of orientation effects (e.g. Best et al. 1995; Scheuer 1995), since the forward edges of the two lobes have different light travel times to the observer. Best et al. (1995) explain the differences in the armlength asymmetries between radio galaxies and quasars in a sample of 3CR sources with orientation differences only, although high expansion velocities of the lobes, up to $0.4 c$, are then required. Since Best et al. find no significant correlation between the parameters describing the arm-length asymmetry and the emission-line asymmetry, they suggest that environmental effects are not necessarily the main cause of the observed asymmetries in 3CR sources, in agreement with Begelmann \& Cioffi (1989).

To investigate if the linear size of the radio source has any influence on the observed asymmetries, we have measured the armlengths of the lobes of all FRII-type sources in the 1-Jy sample. We have calculated the armlengthratio, $Q$, by dividing the length of the longest arm by that of the shortest arm. This yields the fractional separation difference, $x$, which is defined as $x=\frac{Q-1}{Q+1}$ (e.g. Best et al. 1995). The advantage of using $x$, instead of $Q$, is that its range is limited between 0 and 1 . In Table 4 we present the armlengths, $Q, x$, and the references to the data used to measure these parameters.

In Fig. 3a we have plotted a histogram of the fractional separation difference of the GRGs. We have omitted the source B 2043+745 from our GRG sample since it is identified with a quasar and may thus have an extreme orientation; note, however, that this source is very symmetrical $(x=0.01 \pm 0.01)$ and that including it would raise the first bin only by $\sim 0.05$.

As comparison we have plotted the armlength asymmetry distribution of $z<0.3$ (i.e. similar redshift range) FRII-type 3CR radio galaxies with $50<D<1000 \mathrm{kpc}$, for which we have taken the data from Best et al. (1995). We have removed sources smaller than $50 \mathrm{kpc}$ since their asymmetries, if environmental, more reflect the gas distribution inside or close to the host galaxy whereas we are interested in the large-scale environment. There are 27 sources in the 3CR subsample, as compared to 19 FRIItype GRGs.

This comparison is only meaningful if $3 \mathrm{CR}$ sources are in similar gaseous environments as GRGs, and will develop into GRGs provided that their nuclear activity lasts for a long enough time. As yet, there is little detailed knowledge on the difference in the environments of $3 \mathrm{CR}$ and
GRG sources, and on the evolution of radio sources from small to large ones (e.g. Schoenmakers 1999). Radio source evolution models (e.g. Kaiser et al. 1997; Blundell et al. 1999) predict that GRGs must have been much more radio luminous when they were of smaller size, which is not inconsistent with them being $3 \mathrm{CR}$ galaxies at an earlier evolutionary stage (see also Schoenmakers 1999). We will therefore assume that the environments are largely similar for the $>50 \mathrm{kpc} 3 \mathrm{CR}$ sources and the GRGs.

Although the difference in armlength asymmetry between $3 \mathrm{CR}$ radio galaxies and GRGs is small and probably not significant, the GRGs tend to be biased towards higher armlength asymmetries (Fig. 3a). A KolmogorovSmirnoff (K-S) test shows that the two distributions are different at the $95 \%$ confidence level. Note, however, the relatively large (Poissoneous) errors in Fig. 3a, due to the small number of sources in the samples.

We have also measured the bending angle, defined as the angle between the lines connecting the core with the endpoints of the two lobes. The results are presented in Table 4. The distribution of bending angles is plotted in Fig. 3b, together with the values for the $z<0.33 \mathrm{CR}$ galaxies from Best et al. (1995). The distributions are quite similar; a K-S test shows that they do not differ significantly at the $90 \%$ confidence level. Further discussion of these asymmetries will be presented in Sect. 7.3.

\subsection{Flux density and spectral index asymmetries}

We have measured the flux density asymmetry, $R$, of the radio lobes and the spectral index difference, $\Delta \alpha$, between the two lobes. We have defined these parameters such that they increase monotonically with increasing asymmetry, i.e. $R$ is the $325-\mathrm{MHz}$ flux density of the brightest lobe divided by that of the weakest lobe and $\Delta \alpha$ is the spectral index of the lobe with the flattest spectrum minus the spectral index of the lobe with the steepest spectrum, measured between $325 \mathrm{MHz}$ and $10.5 \mathrm{GHz}$. We have searched for correlations between these parameters and the armlength asymmetry parameter $x$ using Spearman rank correlation tests. To avoid spuriously significant correlations as a result of single outliers in the parameter space under investigation, we have omitted, for each of the two parameters being tested, the source with the highest value of that parameter. The results of the correlation tests are presented in Cols. 1-3 of Table 5 .

We find that the only significant correlation is that between $x$ and $\Delta \alpha$, i.e. when a radio source is more asymmetric in armlength, then also the spectral index difference between the lobes is systematically larger. We find that in 15 out of 20 sources the radio lobe with the shortest arm preferentially has a steeper spectrum, although the difference in spectral index between the two sides is $\lesssim 0.1$ for almost all sources (see Fig. 4).

The lobe flux density asymmetry, $R$, is not significantly correlated with either $x$ or $\Delta \alpha$. Still, we find that in 13 
Table 4. Morphological parameters of the radio lobes. Column 1 gives the name of the source. Column 2 indicates which side of the source is named $A$ and $B$ in this table ("N" stands for north, etc.). Columns 3 to 6 give the angular size and the physical size of the lobes. Column 7 gives the asymmetry parameter, $Q$, defined as ratio of the length of the longest lobe to that of the shortest. Column 8 gives the fractional separation parameter, $x$, defined as $x=(Q-1) /(Q+1)$. Column 9 gives the bending angle, $\theta$, of the radio source, defined as the angle between the radio axes of the two lobes

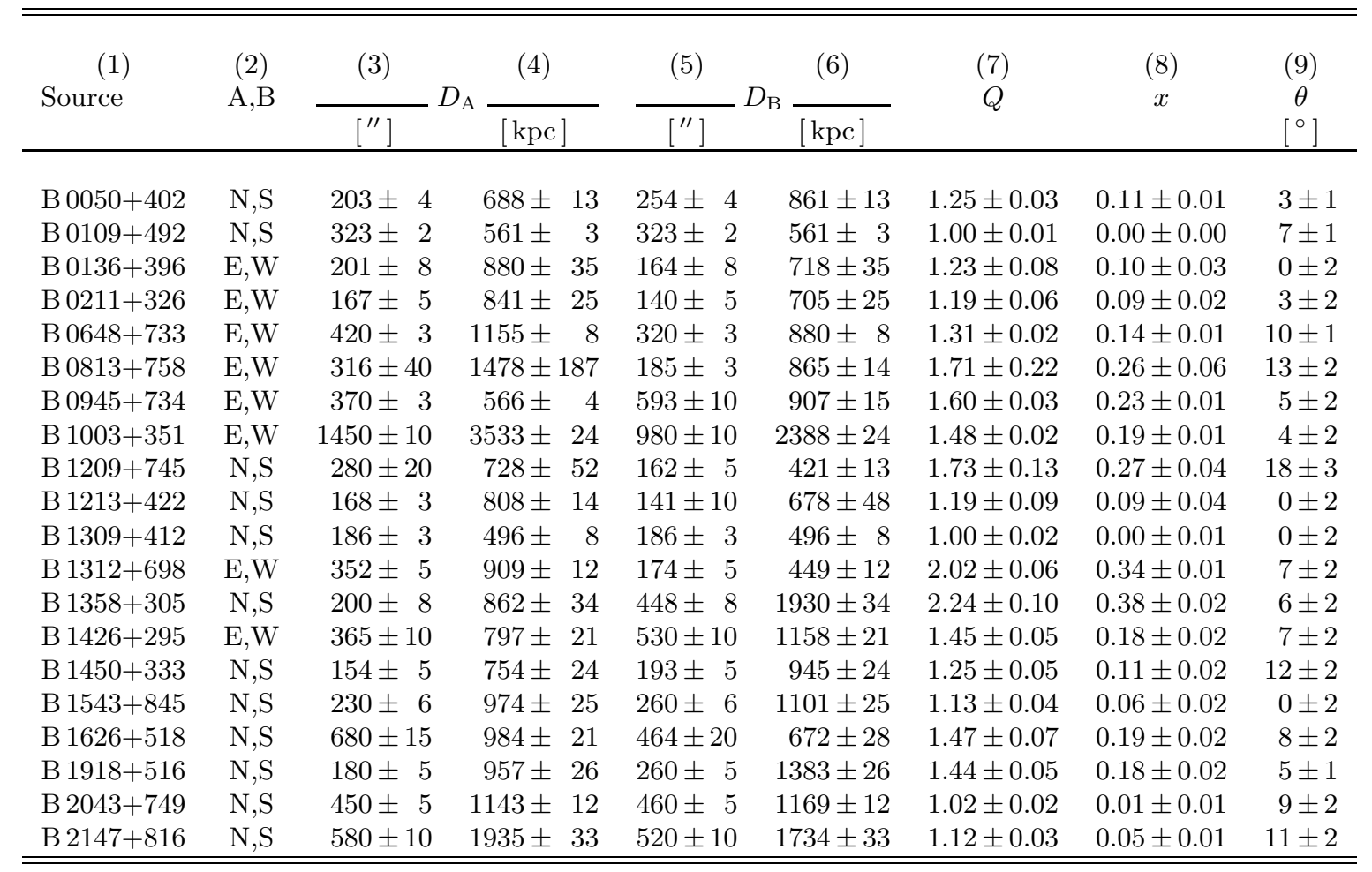

out of 20 sources (i.e. $65 \%$ ) the most luminous radio lobe has the shortest armlength. This is a similar percentage as found in the sample of 3CR sources studied by McCarthy et al. (1991, but see Best et al. 1995) which suggests that this trend does not occur by chance, only.

Since the luminosity of a radio lobe is the lobe volume integrated emissivity, it is perhaps preferable to compare $R$ and $\Delta \alpha$ with the asymmetry in the estimated volume of the radio lobes. Also, if the asymmetries are caused by large-scale environmental inhomogeneities, this will probably affect the dynamical evolution of the lobe as a whole, and not only its forward advance. Therefore, we have investigated the correlation of $R$ and $\Delta \alpha$ with $Q_{V}$, the ratio of the volume of the largest lobe to that of the smallest. See Sect. 6.1 for the method used to estimate the lobe volumes.

The results are presented in the last three columns of Table 5. Although the correlation analysis gives significant results for both $\Delta \alpha$ and $R$ with $Q_{V}$, indicating a correlated increase in asymmetry for these parameters, these results are not very meaningful. We find that in only 12 out of 20 sources the lobe which is smallest in estimated volume has the steepest spectrum. Also, in only 11 out of 20 sources we find that the largest lobe is the brightest. This indicates that the relative volume of a radio lobe has
Table 5. Spearman rank correlation tests between the asymmetry parameters of the GRGs. See the text for details on the definition of the parameters. Columns 1 and 4 give the parameters being tested. Columns 2 and 5 give the correlation coefficients, $r_{\mathrm{s}}$. Columns 3 and 6 give the significance, $s$, of the correlation. The probability of the correlation occurring by chance is $1-s$

\begin{tabular}{rccccc}
\hline \hline$(1)$ & $(2)$ & $(3)$ & $(4)$ & $(5)$ & $(6)$ \\
& $r_{\mathrm{s}}$ & $s$ & & $r_{\mathrm{s}}$ & \multicolumn{1}{c}{$s$} \\
\hline$\Delta \alpha-x$ & 0.657 & 0.997 & $\Delta \alpha-Q_{V}$ & 0.704 & 0.999 \\
$R-x$ & 0.257 & 0.664 & $R-Q_{V}$ & 0.582 & 0.986 \\
$\Delta \alpha-R$ & 0.333 & 0.809 & & & \\
\hline \hline
\end{tabular}

less influence on its relative spectral index than the armlength of the lobe. We will discuss this in more detail in Sect. 7.3.

\section{The advance velocities and ages of the radio lobes}

In this section we derive the ages and velocities of the lobes of the GRGs by making use of the often observed trend 

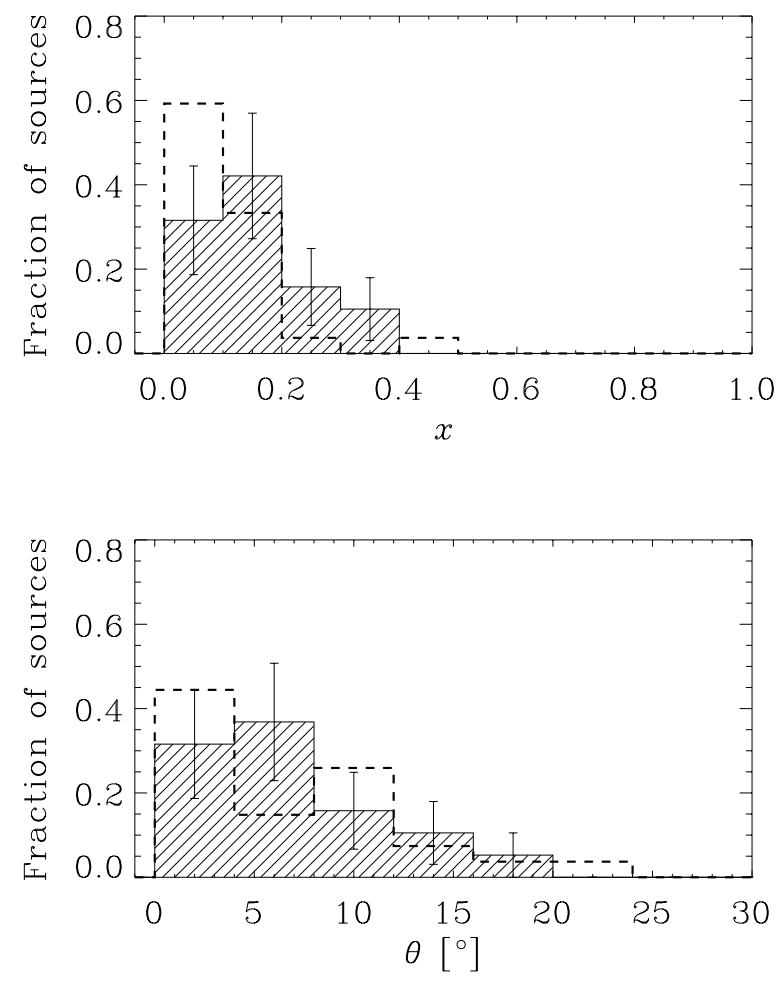

Fig. 3. Plots of the distribution of the fractional separation difference, $x$ (top), and the bending angle, $\theta$ (bottom), of the FRII-type GRGs (hatched area) and of $z<0.3$ and $50<D<1000 \mathrm{kpc}$ powerful 3CR sources from Best et al. (1995; area under dashed line). The error bars assume a poisson distribution of the number of sources in a bin

that the radio spectrum in the radio lobes of FRII-type sources steepens along the line connecting the hotspots with the core. This can be explained as a result of ageing of the radiating particles after they have been accelerated in the hotspots (e.g. Alexander \& Leahy 1987). By fitting spectral ageing models, the spectral index as a function of distance from the hotspot then yields the age as a function of hotspot. From the velocity of the hotspot and the size of the source an estimate of the spectral age of the radio source is obtained. Using ram-pressure equilibrium at the head of the jet, we can then also estimate the density in the ambient medium of the radio lobes.

\subsection{Spectral index profiles}

We have used the WENSS or 325-MHz WSRT maps of Mack et al. (1997), and the 10.5-GHz Effelsberg data to produce profiles of the spectral index, $\alpha_{325}^{10500}$, of the GRGs along their radio axes. We have selected the 15 FRII-type sources with an angular size above $7^{\prime}$, only. This ensures that the radio lobes are covered by several independent beams. We have omitted the source B 1918+516 since it is confused with two unresolved sources in our low

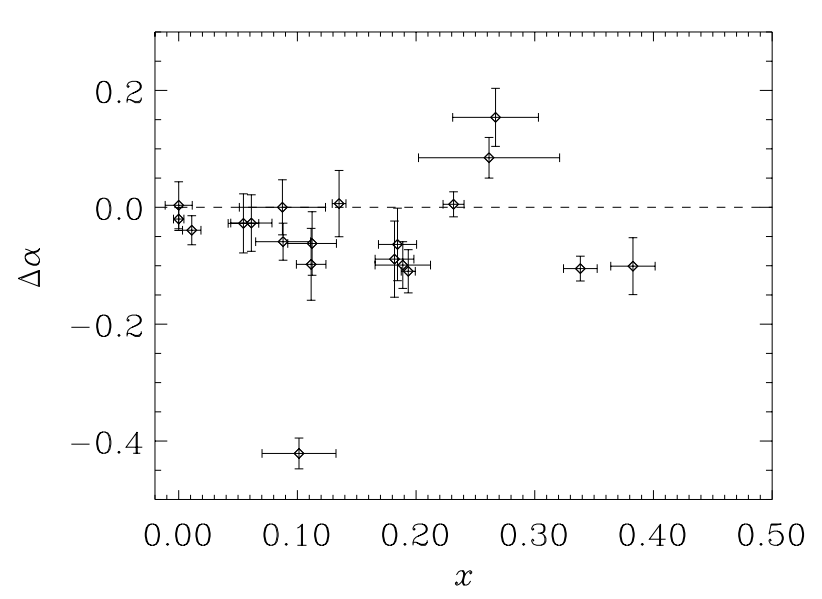

Fig. 4. The difference in spectral index between the longest and the shortest lobe against the armlength asymmetry parameter $x$ of the GRGs. The source with the largest side-to-side difference in spectral index $(\Delta \alpha \approx-0.4)$ is $\mathrm{B} 0136+396$

frequency maps (see Paper I). We have convolved the maps at $325 / 354 \mathrm{MHz}$ to the resolution of the $10.5-\mathrm{GHz}$ Effelsberg maps $\left(69^{\prime \prime} \times 69^{\prime \prime} \mathrm{FWHM}\right)$. In case that the declination of a source is below $+51^{\circ}$, we have convolved both the $325 / 354-\mathrm{MHz}$ and the $10.5-\mathrm{GHz}$ maps to a common resolution of $69^{\prime \prime} \times(54 \cdot \operatorname{cosec} \delta)^{\prime \prime}(\mathrm{FWHM})$. Additionally, for sources with an angular size $\leq 10^{\prime}$ and for which reliable 1.4-GHz NVSS maps are therefore available, we have also made profiles of the spectral indices between $325 / 354 \mathrm{MHz}$ and $1.4 \mathrm{GHz}(\mathrm{B} 0050+402$, B $0813+758$, $\mathrm{B} 1312+698$, B 1543+845), or between $1.4 \mathrm{GHz}$ and 10.5 GHz (B 1358+305; B 0050+402 was omitted here because only few reliable datapoints could be found). For this, the NVSS maps have been convolved to the resolution of either the WENSS or the Effelsberg observations.

We have superposed rectangular boxes on the radio source, with the long side oriented perpendicular to the radio axis and with a width along the radio axes of 0.5 times the FWHM beam size of the convolved map. The whole array of such boxes has been centered on the radio core position. Possible confusing sources have been blanked from the radio maps. In each box, we have integrated the flux density at each frequency and calculated the spectral index of the box from this. If the flux density inside a box was not significant (i.e. $<3 \sigma_{\mathrm{I}}$ ) we used a $3 \sigma_{\mathrm{I}}$ upper flux density limit to limit the spectral index.

The observations we use have been done using different instruments (i.e. VLA, WSRT, 100-m Effelsberg) which may lead to artefacts in the spectral index profiles. In general, these artefacts are most prominent in areas of low signal-to-noise (background level effects) and in areas with large intensity gradients (beam-size effects), such as along the edge of a radio source. Boxes with a low 
signal-to-noise have been presented as limits in the spectral index profiles, so background effects should not be serious in these areas. Large intensity gradients occur mostly at the outer edges of the sources, near the hotspots. Therefore, the outermost point on each side of a source is usually not reliable.

The profiles of the spectral index between $325 / 354 \mathrm{MHz}$ and $10.5 \mathrm{GHz}$ are presented in Fig. 5, those between $325 / 354 \mathrm{MHz}$ and $1.4 \mathrm{GHz}$ in Fig. 6. We find that only a few of the GRGs show a significant steepening of their radio spectra towards the core (e.g. B 2043+749). In other sources, such as B $2147+816$, such a behaviour is not observed. In some cases a likely explanation is that a flat-spectrum radio jet contributes to the extended lobe emission. The source B $1209+745$ is known to have a prominent one-sided jet pointing towards the north (e.g. van Breugel \& Willis 1981), which explains the flat-spectrum "plateau" north of the radio core position in Fig. 5. The same may be true for $\mathrm{B} 1312+698$, which shows jet-like features in an (unpublished) 1.4-GHz WSRT radio map. However, for other sources such a scenario is less probable since no jet-like features appear in any of our radio maps. Among the possible causes for the apparent absence of ageing in these sources can be mixing of the lobe material due to backflows in the lobes, non-uniform magnetic fields and changes in the energy distribution of the accelerated particles during the sources lifetime.

\subsection{The advance velocities of the radio lobes}

We have fitted advance velocities and ages of the radio lobes to the spectral index profiles using the method described in Schoenmakers et al. (1998). In short, this method works as follows: First, we recognize that Inverse Compton scattering of the Microwave Background (MWB) radiation is an important energy loss mechanism of the radiating particles in the lobes of GRGs. Its influence on the energy losses can be described by imposing an additional magnetic field, $B_{\mathrm{MWB}}$, whose energy density equals that of the MWB radiation field, i.e. $B_{\mathrm{MWB}}=3.24(1+z)^{2} \mu \mathrm{G}$, where $z$ is the redshift of the source. In Fig. 7 we have plotted the ratio of the equivalent magnetic field strength of the MWB radiation, $B_{\mathrm{MWB}}$, and the equipartition magnetic field strengths in the radio lobes, $B_{\text {eq }}$, averaged for both lobes, against both redshift and linear size of the GRGs (see also Ishwara-Chandra \& Saikia 1999). See Sect. 6 for the calculation of the equipartition magnetic field strength. We find that in all GRGs $B_{\text {eq }} \lesssim B_{\mathrm{MWB}}$, which indicates that the IC-scattering process dominates the energy losses of the radio lobes. If we neglect energy losses resulting from the expansion of the lobes, then the radiating particles loose energy only due to synchrotron radiation and IC scattering of MWB photons. In this case, the radiative lifetime of particles is maximized when the internal magnetic field strength of the lobe $B_{\text {int }}=B_{\mathrm{MWB}} / \sqrt{3}$ (e.g. van der Laan \& Perola 1969). In other words, for a given time since the last acceleration of the radiating particles, this magnetic field strength gives the least amount of spectral steepening due to radiation and IC scattering. Using this value for the internal magnetic field strength therefore provides an upper limit to the spectral age of a radio source.

We have derived the velocities and ages of the radio lobes using both the equipartition magnetic field strength (see Table 7) and the magnetic field strength which gives the maximum age $\left(B_{\mathrm{MWB}} / \sqrt{3}\right)$. We have calculated the velocities and ages for two ageing models: The JaffePerola (JP) and the Continuous Injection (CI) models (see Schoenmakers et al. 1998, and references therein, for details). These are the extreme cases in the sense that the JP model gives the largest change in spectral index for a given age, whereas the CI model gives the smallest change. We find that in many cases our data are not good enough to decide which model is better applicable. It can be expected, however, that this is the JP model unless reacceleration of the particles in the lobes is important. Last, we assume that the advance velocity of the radio lobes, the injection spectral index of the radiating particles and the magnetic field strength in the lobes have been constant during the life-time of the radio source, and that the magnetic field strength is uniform throughout the lobe.

The velocity we find with our method is the separation velocity between the head of the lobe and the material flowing back in the lobe. This backflow may be important in very powerful radio sources (e.g. Liu et al. 1992; Scheuer 1995), but whether this is also the case in GRGs is not clear. Some of our sources have radio lobes which do not cover the whole area between the hotspot and the radio core. Although this may be caused by the increased effect of spectral ageing at a large distance from the hotspot, it may also indicate that backflows are not important in these radio lobes. Although the issue is far from clear, we will assume for the remainder of this discussion that backflows are unimportant in the lobes of the GRGs.

Many of our sources either do not show the expected ageing signature, i.e. a steepening of the spectrum towards the radio core, or have too few (less than five) or too poorly determined datapoints to provide a meaningful constraint for the fitting process (see Figs. 5 and 6). Only seven sources have spectral index profiles that we could use to fit the velocities and ages. These are B $0109+492$, B 0813+758 (between 354 and $1400 \mathrm{MHz}$ ), B 1003+351 (western lobe only), B 1209+745 (between 354 and $1400 \mathrm{MHz}), \mathrm{B} 1312+698$, B $1543+845$ and B $2043+749$. The results for these are presented in Table 6 , and the model fits in Fig. 8. The plots in Fig. 8 have been made for the case that $B_{\text {int }}=B_{\mathrm{MWB}} / \sqrt{3}$. In the table we present for each lobe the best fit velocity, $v$, and injection spectral index, $\alpha_{\text {inj }}$, and the reduced $\chi^{2}$ of the fit. In case of the source B $2043+749$ we are able to fit the spectral 

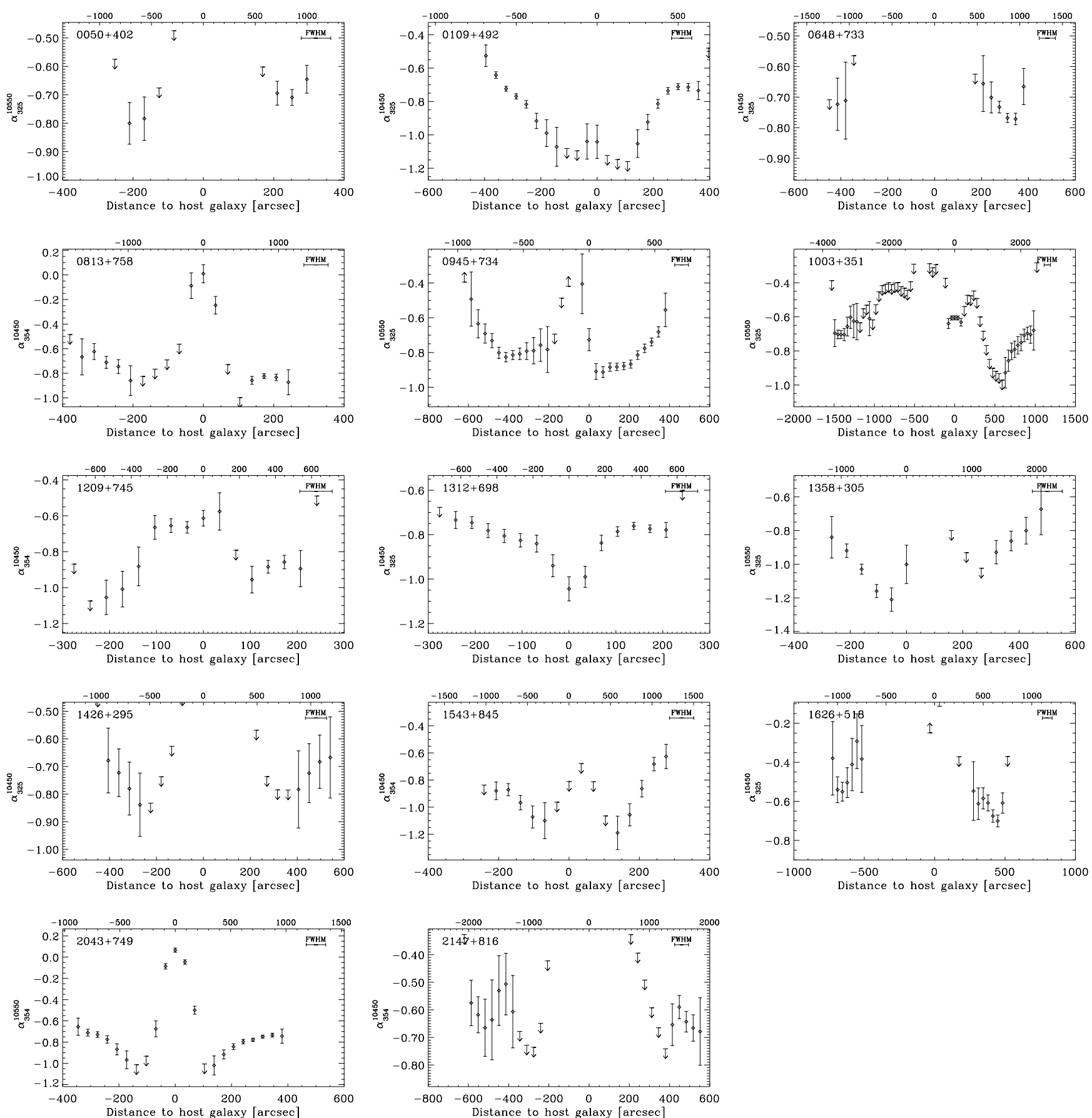

Fig. 5. Profiles of the spectral index distribution along the major axes of the radio sources, between 325 (or 354 ) MHz and $10.5 \mathrm{GHz}$. The zero point on the $x$-axis is the position of the radio core. The numbers along the upper axis denote the distance from the radio core in units of kpc. Negative values are on that side of the source which is mentioned first in column two of Table 7

index profile of the southern lobe using either five or seven spectral index points. The results are significantly different and we therefore present them for both these cases. The values of the reduced $\chi^{2}$ presented in Table 6 are almost all smaller than unity. We believe that this is largely due to our conservative error estimates in the spectral indices.

We find that the advance velocities of the heads of the lobes of the GRG are in the range of $0.01 c-0.1 c$, with an average value of $\sim 0.04 c$. The many sources that could not 

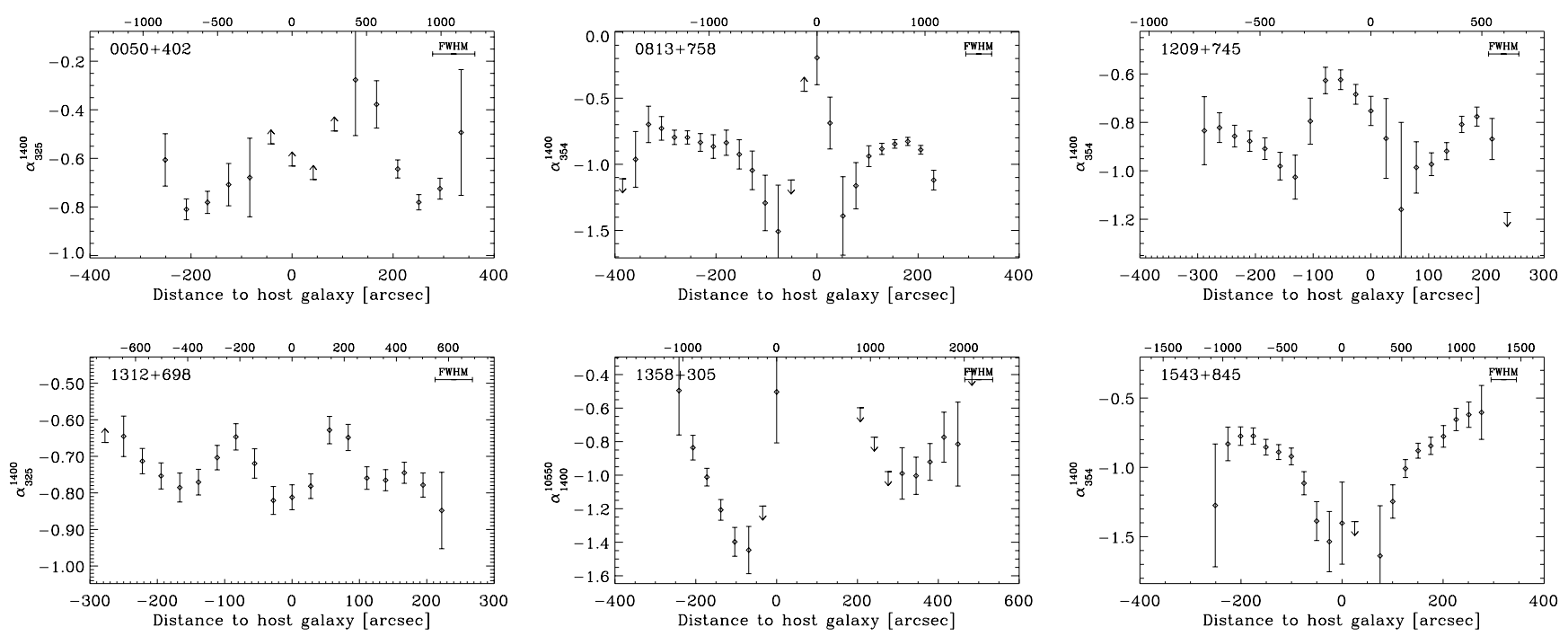

Fig. 6. Profiles of the spectral index distribution along the major axes of the sources smaller than $10^{\prime}$, between 325 (or 354 ) MHz and $1.4 \mathrm{GHz}$ (except for B 1358+305, which is between 1.4 and $10.5 \mathrm{GHz}$ ). The axes have been annotated similarly as in Fig. 5
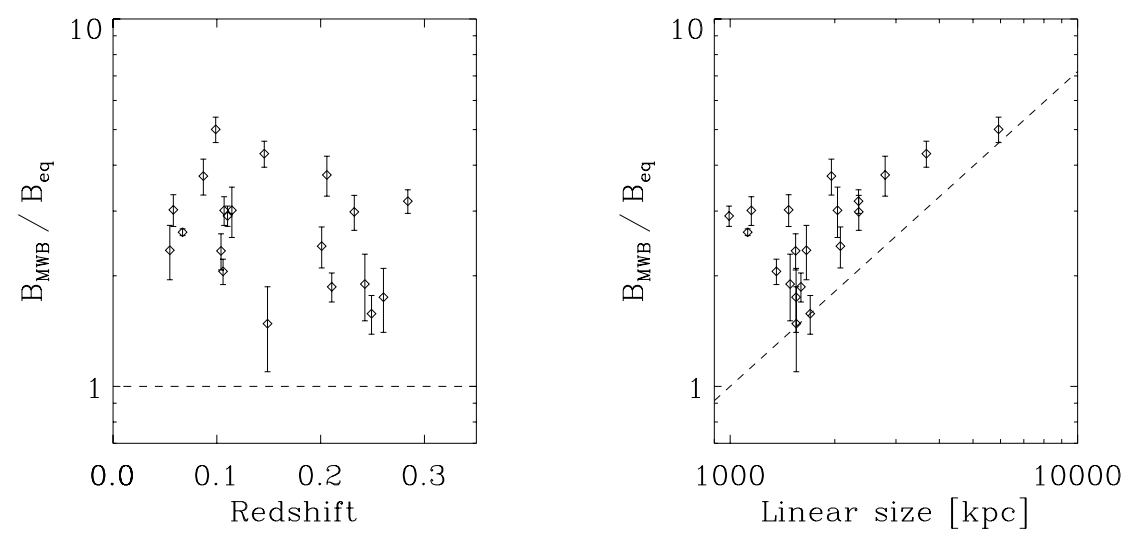

Fig. 7. The ratio of the equivalent magnetic field strength of the microwave background radiation, $B_{\mathrm{MwB}}$, to the averaged equipartition magnetic field strengths, $B_{\text {eq }}$, in the lobes of the GRGs against redshift (left) and projected linear size (right) of the radio source. The dashed line in the right plot indicates the expected behaviour of $B_{\mathrm{MWB}} / B_{\text {eq }}$ for increasing linear size of a source with constant radio power and redshift $\left(B_{\text {eq }} \propto V^{-2 / 7} \propto D^{-6 / 7}\right.$, where $V$ and $D$ are the volume and linear size of the radio source, respectively). This line only illustrates that the apparent correlation in this diagram is due to selection effects

be fitted using our method clearly question the validity of our method and the results. If some of the mechanisms that prevent the presence of a clear ageing signature in all these sources are also at work in the seven sources that we were able to find the age for, then the velocities that we have obtained are only upper limits to the true velocities. They indicate a general trend, though, that the advance velocities of the lobes of GRGs are below $0.1 c$.

\subsection{The spectral ages of GRGs}

The method to find the velocities of the lobes which we employed in Sect. 5.2 also yields the spectral age of the radio source. Using the lobe advance velocities in Table 6 and the known armlengths of the lobes (Table 4), we have calculated the lobe ages. We have calculated the ages for each of the two spectral ageing models and for both the equipartition magnetic field strength and the one that should maximize the age of the lobe, $B_{\mathrm{MWB}} / \sqrt{3}$.

In Table 6 we present the spectral ages of the lobes of the seven GRGs in our sample for which we could find the lobe velocities. In general, the differences in the spectral age for the two values of the magnetic field strength are small. In reality the age of the two lobes of a single source should be equal. In all sources where we could fit both lobes, we indeed find that the ages are close to each other. In the southern lobe of the source B $2043+749$, which we 
Table 6. Results of the spectral aging analysis. The top part of the table presents the results for an internal magnetic field strength equal to $B_{\mathrm{MWB}} / \sqrt{3}$, which should give the maximum age; the bottom part is for an internal field strength equal to the average equipartition field strength in the two lobes. Column 1 gives the name of the source and the component. Column 2 gives the number of spectral index points used in the fitting. Columns 3 to 5 give the velocity, $v$, in units of $c$, the injection spectral index, $\alpha_{\text {inj }}$ and the reduced $\chi^{2}$ of the fit using the CI model. Columns 6 to 8 give the same for the JP model. Columns 9 and 10 give the age of the lobes resulting from the fitted velocities and the size of the lobes, for each of the two models

\begin{tabular}{|c|c|c|c|c|c|c|c|c|c|c|}
\hline \multirow{2}{*}{\multicolumn{2}{|c|}{$\begin{array}{c}(1) \\
\text { Source }\end{array}$}} & \multirow[t]{2}{*}{$(2)$} & \multirow{2}{*}{$\begin{array}{l}(3) \\
v_{\mathrm{CI}} \\
{[c]}\end{array}$} & (4) & \multirow{2}{*}{$\begin{array}{c}(5) \\
\chi_{\mathrm{red}}^{2}\end{array}$} & \multirow{2}{*}{$\begin{array}{l}(6) \\
v_{\mathrm{JP}} \\
{[c]}\end{array}$} & \multirow{2}{*}{$\begin{array}{c}(7) \\
\alpha_{\mathrm{inj}, \mathrm{JP}}\end{array}$} & \multirow{2}{*}{$\begin{array}{c}(8) \\
\chi_{\mathrm{red}}^{2}\end{array}$} & \multirow{2}{*}{\multicolumn{2}{|c|}{$\begin{array}{l}(9) \\
t_{\mathrm{CI}} \\
\quad\left[10^{7} \mathrm{yr}\right]\end{array}$}} \\
\hline & & & & $\alpha_{\mathrm{inj}, \mathrm{CI}}$ & & & & & & \\
\hline В $0109+492$ & $\begin{array}{l}\mathrm{N} \\
\mathrm{S}\end{array}$ & $\begin{array}{l}6 \\
5\end{array}$ & $\begin{array}{l}0.014 \pm 0.001 \\
0.014 \pm 0.001\end{array}$ & $\begin{array}{l}-0.72 \pm 0.01 \\
-0.69 \pm 0.01\end{array}$ & $\begin{array}{l}0.38 \\
0.68\end{array}$ & $\begin{array}{l}0.040 \pm 0.001 \\
0.029 \pm 0.001\end{array}$ & $\begin{array}{l}-0.74 \pm 0.01 \\
-0.69 \pm 0.01\end{array}$ & $\begin{array}{l}0.46 \\
0.86\end{array}$ & $\begin{array}{l}13.1 \pm 0.9 \\
13.1 \pm 0.9\end{array}$ & $\begin{array}{l}4.6 \pm 0.1 \\
6.2 \pm 0.2\end{array}$ \\
\hline B $0813+758$ & $\begin{array}{l}\mathrm{E} \\
\mathrm{W}\end{array}$ & $\begin{array}{c}11 \\
6\end{array}$ & $\begin{array}{l}0.046 \pm 0.001 \\
0.032 \pm 0.001\end{array}$ & $\begin{array}{l}-0.71 \pm 0.03 \\
-0.81 \pm 0.04\end{array}$ & $\begin{array}{l}0.49 \\
0.55\end{array}$ & $\begin{array}{l}0.094 \pm 0.001 \\
0.061 \pm 0.001\end{array}$ & $\begin{array}{l}-0.71 \pm 0.03 \\
-0.81 \pm 0.04\end{array}$ & $\begin{array}{l}0.34 \\
0.43\end{array}$ & $\begin{array}{r}10.5 \pm 1.3 \\
8.8 \pm 0.3\end{array}$ & $\begin{array}{l}5.1 \pm 0.7 \\
4.6 \pm 0.1\end{array}$ \\
\hline B $1003+351$ & $\mathrm{~W}$ & 8 & $0.059 \pm 0.002$ & $-0.70 \pm 0.02$ & 0.09 & $0.119 \pm 0.002$ & $-0.70 \pm 0.02$ & 0.06 & $13.1 \pm 0.4$ & $6.5 \pm 0.1$ \\
\hline B $1209+745$ & $\begin{array}{l}\mathrm{N} \\
\mathrm{S}\end{array}$ & $\begin{array}{l}6 \\
6\end{array}$ & $\begin{array}{l}0.017 \pm 0.001 \\
0.011 \pm 0.001\end{array}$ & $\begin{array}{l}-0.81 \pm 0.03 \\
-0.78 \pm 0.03\end{array}$ & $\begin{array}{l}0.07 \\
0.34\end{array}$ & $\begin{array}{l}0.033 \pm 0.001 \\
0.024 \pm 0.001\end{array}$ & $\begin{array}{l}-0.81 \pm 0.03 \\
-0.79 \pm 0.03\end{array}$ & $\begin{array}{l}0.09 \\
0.47\end{array}$ & $\begin{array}{l}14.0 \pm 1.3 \\
12.5 \pm 1.2\end{array}$ & $\begin{array}{l}7.2 \pm 0.6 \\
5.7 \pm 0.3\end{array}$ \\
\hline В $1312+698$ & $\begin{array}{l}\mathrm{E} \\
\mathrm{W}\end{array}$ & $\begin{array}{l}6 \\
5\end{array}$ & $\begin{array}{l}0.037 \pm 0.002 \\
0.024 \pm 0.002\end{array}$ & $\begin{array}{l}-0.71 \pm 0.02 \\
-0.75 \pm 0.02\end{array}$ & $\begin{array}{l}0.42 \\
0.96\end{array}$ & $\begin{array}{l}0.103 \pm 0.002 \\
0.056 \pm 0.002\end{array}$ & $\begin{array}{l}-0.74 \pm 0.02 \\
-0.76 \pm 0.02\end{array}$ & $\begin{array}{l}0.17 \\
0.57\end{array}$ & $\begin{array}{l}8.0 \pm 0.4 \\
6.1 \pm 0.5\end{array}$ & $\begin{array}{l}2.9 \pm 0.1 \\
2.6 \pm 0.1\end{array}$ \\
\hline B $1543+845$ & $\begin{array}{l}\mathrm{N} \\
\mathrm{S}\end{array}$ & $\begin{array}{l}7 \\
8\end{array}$ & $\begin{array}{l}0.025 \pm 0.001 \\
0.021 \pm 0.001\end{array}$ & $\begin{array}{l}-0.75 \pm 0.04 \\
-0.65 \pm 0.04\end{array}$ & $\begin{array}{l}1.17 \\
0.96\end{array}$ & $\begin{array}{l}0.051 \pm 0.001 \\
0.045 \pm 0.001\end{array}$ & $\begin{array}{l}-0.74 \pm 0.04 \\
-0.65 \pm 0.03\end{array}$ & $\begin{array}{l}0.58 \\
0.34\end{array}$ & $\begin{array}{l}12.7 \pm 0.7 \\
17.2 \pm 1.3\end{array}$ & $\begin{array}{l}6.2 \pm 0.2 \\
8.0 \pm 0.3\end{array}$ \\
\hline B $2043+749$ & $\begin{array}{l}\mathrm{N} \\
\mathrm{S} \\
\mathrm{S} \\
\end{array}$ & $\begin{array}{l}5 \\
5 \\
7 \\
\end{array}$ & $\begin{array}{l}0.029 \pm 0.001 \\
0.030 \pm 0.002 \\
0.059 \pm 0.002 \\
\end{array}$ & $\begin{array}{l}-0.70 \pm 0.02 \\
-0.77 \pm 0.02 \\
-0.74 \pm 0.01 \\
\end{array}$ & $\begin{array}{l}0.27 \\
0.52 \\
0.83 \\
\end{array}$ & $\begin{array}{l}0.057 \pm 0.001 \\
0.060 \pm 0.002 \\
0.112 \pm 0.002 \\
\end{array}$ & $\begin{array}{l}-0.70 \pm 0.02 \\
-0.77 \pm 0.02 \\
-0.74 \pm 0.01 \\
\end{array}$ & $\begin{array}{l}0.07 \\
0.42 \\
0.55 \\
\end{array}$ & $\begin{array}{l}8.6 \pm 0.3 \\
8.5 \pm 0.5 \\
4.3 \pm 0.2 \\
\end{array}$ & $\begin{array}{l}4.3 \pm 0.1 \\
4.3 \pm 0.2 \\
2.3 \pm 0.1 \\
\end{array}$ \\
\hline В $0109+492$ & $\begin{array}{l}\mathrm{N} \\
\mathrm{S}\end{array}$ & $\begin{array}{l}6 \\
5\end{array}$ & $\begin{array}{l}0.016 \pm 0.001 \\
0.016 \pm 0.001\end{array}$ & $\begin{array}{l}-0.72 \pm 0.01 \\
-0.70 \pm 0.02\end{array}$ & $\begin{array}{l}0.39 \\
0.73\end{array}$ & $\begin{array}{l}0.045 \pm 0.001 \\
0.033 \pm 0.001\end{array}$ & $\begin{array}{l}-0.73 \pm 0.01 \\
-0.70 \pm 0.01\end{array}$ & $\begin{array}{l}0.51 \\
0.24\end{array}$ & $\begin{array}{l}11.4 \pm 0.7 \\
11.4 \pm 0.7\end{array}$ & $\begin{array}{l}4.1 \pm 0.1 \\
5.5 \pm 0.2\end{array}$ \\
\hline В $0813+758$ & $\begin{array}{l}\mathrm{E} \\
\mathrm{W}\end{array}$ & $\begin{array}{c}11 \\
6\end{array}$ & $\begin{array}{l}0.054 \pm 0.002 \\
0.037 \pm 0.001\end{array}$ & $\begin{array}{l}-0.71 \pm 0.03 \\
-0.81 \pm 0.03\end{array}$ & $\begin{array}{l}0.48 \\
0.55\end{array}$ & $\begin{array}{l}0.110 \pm 0.002 \\
0.071 \pm 0.001\end{array}$ & $\begin{array}{l}-0.71 \pm 0.03 \\
-0.81 \pm 0.04\end{array}$ & $\begin{array}{l}0.34 \\
0.44\end{array}$ & $\begin{array}{l}8.9 \pm 1.2 \\
7.7 \pm 0.4\end{array}$ & $\begin{array}{l}4.4 \pm 0.5 \\
4.0 \pm 0.2\end{array}$ \\
\hline B $1003+351$ & $\mathrm{~W}$ & 8 & $0.091 \pm 0.002$ & $-0.69 \pm 0.02$ & 0.13 & $0.173 \pm 0.002$ & $-0.70 \pm 0.02$ & 0.06 & $8.5 \pm 0.2$ & $4.5 \pm 0.1$ \\
\hline B $1209+745$ & $\begin{array}{l}\mathrm{N} \\
\mathrm{S}\end{array}$ & $\begin{array}{l}6 \\
6\end{array}$ & $\begin{array}{l}0.019 \pm 0.001 \\
0.013 \pm 0.001\end{array}$ & $\begin{array}{l}-0.81 \pm 0.03 \\
-0.78 \pm 0.03\end{array}$ & $\begin{array}{l}0.08 \\
0.37\end{array}$ & $\begin{array}{l}0.038 \pm 0.001 \\
0.028 \pm 0.001\end{array}$ & $\begin{array}{l}-0.81 \pm 0.03 \\
-0.79 \pm 0.02\end{array}$ & $\begin{array}{l}0.09 \\
0.48\end{array}$ & $\begin{array}{l}12.5 \pm 1.1 \\
10.6 \pm 0.9\end{array}$ & $\begin{array}{l}6.2 \pm 0.5 \\
4.9 \pm 0.2\end{array}$ \\
\hline B $1312+698$ & $\begin{array}{l}\mathrm{E} \\
\mathrm{W}\end{array}$ & $\begin{array}{l}6 \\
5\end{array}$ & $\begin{array}{l}0.038 \pm 0.002 \\
0.025 \pm 0.002\end{array}$ & $\begin{array}{l}-0.71 \pm 0.02 \\
-0.75 \pm 0.02\end{array}$ & $\begin{array}{l}0.41 \\
0.97\end{array}$ & $\begin{array}{l}0.105 \pm 0.002 \\
0.055 \pm 0.002\end{array}$ & $\begin{array}{l}-0.74 \pm 0.02 \\
-0.75 \pm 0.02\end{array}$ & $\begin{array}{l}0.25 \\
0.63\end{array}$ & $\begin{array}{l}7.8 \pm 0.4 \\
5.8 \pm 0.5\end{array}$ & $\begin{array}{l}2.8 \pm 0.1 \\
2.7 \pm 0.1\end{array}$ \\
\hline B $1543+845$ & $\begin{array}{l}\mathrm{N} \\
\mathrm{S}\end{array}$ & $\begin{array}{l}7 \\
8\end{array}$ & $\begin{array}{l}0.027 \pm 0.001 \\
0.022 \pm 0.001\end{array}$ & $\begin{array}{l}-0.75 \pm 0.03 \\
-0.65 \pm 0.04\end{array}$ & $\begin{array}{l}1.17 \\
0.96\end{array}$ & $\begin{array}{l}0.055 \pm 0.001 \\
0.049 \pm 0.001\end{array}$ & $\begin{array}{l}-0.74 \pm 0.03 \\
-0.65 \pm 0.03\end{array}$ & $\begin{array}{l}0.58 \\
0.34\end{array}$ & $\begin{array}{l}11.8 \pm 0.7 \\
16.4 \pm 1.3\end{array}$ & $\begin{array}{l}5.8 \pm 0.2 \\
7.3 \pm 0.3\end{array}$ \\
\hline B $2043+749$ & $\begin{array}{l}\mathrm{N} \\
\mathrm{S} \\
\mathrm{S}\end{array}$ & $\begin{array}{l}5 \\
5 \\
7\end{array}$ & $\begin{array}{l}0.034 \pm 0.001 \\
0.036 \pm 0.002 \\
0.047 \pm 0.002\end{array}$ & $\begin{array}{l}-0.70 \pm 0.02 \\
-0.76 \pm 0.02 \\
-0.71 \pm 0.01\end{array}$ & $\begin{array}{l}0.34 \\
0.63 \\
0.77\end{array}$ & $\begin{array}{l}0.067 \pm 0.001 \\
0.071 \pm 0.002 \\
0.093 \pm 0.002\end{array}$ & $\begin{array}{l}-0.70 \pm 0.02 \\
-0.76 \pm 0.02 \\
-0.71 \pm 0.01\end{array}$ & $\begin{array}{l}0.15 \\
0.51 \\
0.64\end{array}$ & $\begin{array}{l}7.3 \pm 0.2 \\
7.1 \pm 0.4 \\
5.4 \pm 0.3\end{array}$ & $\begin{array}{l}3.7 \pm 0.1 \\
3.6 \pm 0.2 \\
2.7 \pm 0.2\end{array}$ \\
\hline
\end{tabular}

have fitted using both five and seven spectral index points, the best agreement between the ages of the two lobes is found for the fit which uses five spectral points only.

The spectral ages we find all lie in the range between 30 and $150 \mathrm{Myr}$, depending on the spectral ageing model and, to a lesser degree, on the used value of the internal magnetic fieldstrength. Also, they are comparable to those found for other Giant radio sources, using similar techniques, e.g. $40 \mathrm{Myr}$ for B 0136+396 (Hine 1979) and B 0821+695 (Lacy et al. 1993), $180 \mathrm{Myr}$ in B 0319-454 (Saripalli et al. 1994), 140 Myr in B 0313+683
(Schoenmakers et al. 1998). We find an average age of 80 Myr.

\section{The energy densities and pressures of the radio lobes}

The pressure in the bridges of radio lobes of FRII-type sources are higher than that of their environment (e.g. Subrahmanyan \& Saripalli 1993; Subrahmanyan et al. 1996). Radio source evolution models predict that the pressure in the lobes decreases with increasing source 

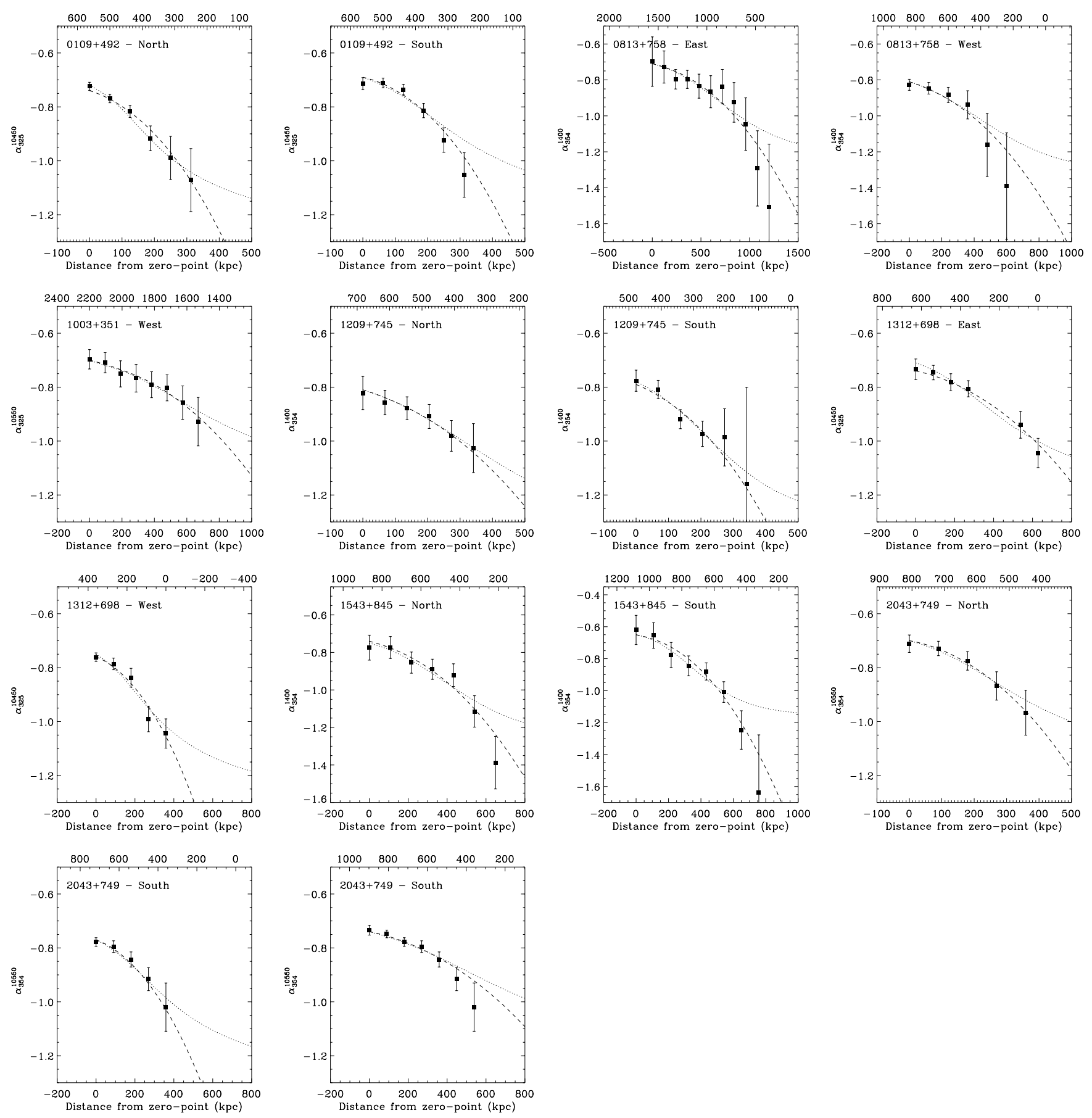

Fig. 8. The results of the spectral ageing analyses. The plotted points are the spectral index points used for the fit in each lobe of the seven sources and the lines are the best fits for the two ageing models. The dotted line represents the CI model and the dashed line the JP model. The numbers along lower axes denote the distance in kpc from the point where we assume that the age is zero. The numbers along the top axis indicate the distance from the host galaxy in kpc. The results plotted here are those using an internal magnetic field strength of $B_{\mathrm{MWB}} / \sqrt{3}$, which should yield the maximum age. For the source B $1003+351$ only the western lobe could be used for fitting. For the southern lobe of B $2043+749$ we present two fits, one using seven and one using five spectral index points, since these give quite different results (see Table 6)

size (e.g. Kaiser \& Alexander 1997), so that the lobes of large radio sources should be closer to pressure equilibrium with their environment. Since GRGs are the largest radio sources known, they are best suited to constrain the pressure in the ambient medium. In this section we investigate the energy densities in the lobe of the FRII-type 
Table 7. The equipartition magnetic field strengths and energy densities of the two lobes of the FRII-type sources in the 1-Jy sample. We have omitted the source $0309+411$ since it is strongly core dominated which did not allow an accurate measurement. Column 1 gives the name of the source. Column 2 gives the sidedness indicator of the lobes "A" and "B", where "N" stands for north, "E" for east, etc. Columns 3 to 6 give the lengths, $l$, and widths, $w$, of the lobes (note, that $l$ only gives the part of the lobe from which radio emission has been detected). Column 7 gives the reference for the observations we used to determine $l$ and $w$. Columns 8 and 9 give the equipartition magnetic field strength and Cols. 10 and 11 give the equipartition energy densities

\begin{tabular}{|c|c|c|c|c|c|c|c|c|c|c|}
\hline (1) & (2) & (3) & (4) & (5) & (6) & $(7)$ & \multirow{2}{*}{\multicolumn{2}{|c|}{$[\mu \mathrm{G}]$}} & $(10)$ & (11) \\
\hline Source & $\mathrm{A}, \mathrm{B}$ & $\begin{array}{l}l_{\mathrm{A}} \\
{\left[{ }^{\prime \prime}\right]}\end{array}$ & $\begin{array}{l}w_{\mathrm{A}} \\
{\left[{ }^{\prime \prime}\right]}\end{array}$ & $\begin{array}{l}l_{\mathrm{B}} \\
{\left[{ }^{\prime \prime}\right]}\end{array}$ & $\begin{array}{l}w_{\mathrm{B}} \\
{\left[{ }^{\prime \prime}\right]}\end{array}$ & & & & $\begin{array}{l}u_{\text {eq, }, \mathrm{A}} \\
{\left[10^{-14} \mathrm{erg}\right.}\end{array}$ & $\begin{array}{c}\begin{array}{l}u_{\text {eq, }}, \\
\left.\mathrm{cm}^{-3}\right]\end{array} \\
\end{array}$ \\
\hline B $0050+402$ & $\mathrm{~N}, \mathrm{~S}$ & $120 \pm 10$ & $30 \pm 10$ & $160 \pm 10$ & $40 \pm 10$ & 1 & $3.05 \pm 0.93$ & $2.72 \pm 0.57$ & $86.3 \pm 37.3$ & $68.7 \pm 20.2$ \\
\hline В $0109+492$ & $\mathrm{~N}, \mathrm{~S}$ & $323 \pm 2$ & $210 \pm 5$ & $323 \pm 2$ & $210 \pm 5$ & 2 & $1.44 \pm 0.03$ & $1.36 \pm 0.03$ & $19.3 \pm 0.6$ & $17.3 \pm 0.5$ \\
\hline В $0136+396$ & $\mathrm{E}, \mathrm{W}$ & $201 \pm 8$ & $90 \pm 10$ & $164 \pm 8$ & $90 \pm 10$ & 3 & $1.81 \pm 0.16$ & $3.28 \pm 0.30$ & $30.5 \pm 3.9$ & $99.7 \pm 12.8$ \\
\hline B $0211+326$ & $\mathrm{E}, \mathrm{W}$ & $167 \pm 5$ & $40 \pm 10$ & $140 \pm 5$ & $40 \pm 10$ & 1 & $2.85 \pm 0.56$ & $3.03 \pm 0.60$ & $75.6 \pm 21.0$ & $85.3 \pm 23.9$ \\
\hline B $0648+733$ & $\mathrm{E}, \mathrm{W}$ & $200 \pm 30$ & $130 \pm 20$ & $200 \pm 20$ & $130 \pm 20$ & 4 & $0.98 \pm 0.18$ & $1.69 \pm 0.24$ & $9.0 \pm 2.3$ & $26.4 \pm 5.3$ \\
\hline В $0813+758$ & $\mathrm{E}, \mathrm{W}$ & $250 \pm 40$ & $100 \pm 10$ & $140 \pm 10$ & $100 \pm 10$ & 4 & $1.74 \pm 0.21$ & $1.56 \pm 0.15$ & $28.0 \pm 4.8$ & $22.6 \pm 3.0$ \\
\hline В $0945+734$ & $\mathrm{E}, \mathrm{W}$ & $370 \pm 3$ & $385 \pm 10$ & $440 \pm 10$ & $250 \pm 50$ & 2 & $0.96 \pm 0.02$ & $1.43 \pm 0.22$ & $8.6 \pm 0.3$ & $19.1 \pm 4.0$ \\
\hline B $1003+351$ & $\mathrm{E}, \mathrm{W}$ & $1450 \pm 10$ & $160 \pm 20$ & $980 \pm 10$ & $340 \pm 30$ & 5 & $0.86 \pm 0.08$ & $0.70 \pm 0.04$ & $6.9 \pm 0.9$ & $4.6 \pm 0.4$ \\
\hline B $1209+745$ & $\mathrm{~N}, \mathrm{~S}$ & $150 \pm 30$ & $170 \pm 10$ & $112 \pm 10$ & $180 \pm 10$ & 2 & $1.29 \pm 0.14$ & $1.35 \pm 0.09$ & $15.3 \pm 2.4$ & $16.9 \pm 1.6$ \\
\hline B $1213+422$ & $\mathrm{~N}, \mathrm{~S}$ & $168 \pm 3$ & $40 \pm 10$ & $141 \pm 10$ & $40 \pm 10$ & 6 & $2.57 \pm 0.50$ & $2.70 \pm 0.58$ & $61.2 \pm 16.9$ & $67.7 \pm 20.5$ \\
\hline B $1309+412$ & $\mathrm{~N}, \mathrm{~S}$ & $186 \pm 3$ & $120 \pm 10$ & $186 \pm 3$ & $120 \pm 10$ & 3 & $1.44 \pm 0.09$ & $1.31 \pm 0.08$ & $19.2 \pm 1.7$ & $15.8 \pm 1.4$ \\
\hline B $1312+698$ & $\mathrm{E}, \mathrm{W}$ & $352 \pm 5$ & $95 \pm 10$ & $174 \pm 5$ & $95 \pm 10$ & 7 & $1.53 \pm 0.12$ & $2.32 \pm 0.19$ & $21.8 \pm 2.3$ & $50.1 \pm 5.7$ \\
\hline B $1358+305$ & $\mathrm{~N}, \mathrm{~S}$ & $200 \pm 8$ & $210 \pm 20$ & $180 \pm 30$ & $130 \pm 20$ & 8 & $1.26 \pm 0.10$ & $1.24 \pm 0.21$ & $14.7 \pm 1.6$ & $14.4 \pm 3.5$ \\
\hline B $1426+295$ & $\mathrm{E}, \mathrm{W}$ & $365 \pm 10$ & $140 \pm 20$ & $350 \pm 20$ & $115 \pm 15$ & 9 & $0.99 \pm 0.11$ & $1.06 \pm 0.12$ & $9.1 \pm 1.4$ & $10.4 \pm 1.7$ \\
\hline B $1450+333$ & $\mathrm{~N}, \mathrm{~S}$ & $100 \pm 10$ & $40 \pm 5$ & $120 \pm 10$ & $40 \pm 5$ & 6 & $3.71 \pm 0.45$ & $2.70 \pm 0.32$ & $127.5 \pm 21.8$ & $67.8 \pm 11.5$ \\
\hline B $1543+845$ & $\mathrm{~N}, \mathrm{~S}$ & $170 \pm 20$ & $60 \pm 5$ & $185 \pm 20$ & $60 \pm 10$ & 4 & $2.03 \pm 0.20$ & $1.85 \pm 0.29$ & $38.3 \pm 5.4$ & $31.7 \pm 7.1$ \\
\hline B $1626+518$ & $\mathrm{~N}, \mathrm{~S}$ & $360 \pm 30$ & $45 \pm 10$ & $320 \pm 30$ & $150 \pm 20$ & 10 & $1.84 \pm 0.36$ & $1.24 \pm 0.15$ & $31.3 \pm 8.8$ & $14.2 \pm 2.5$ \\
\hline B $1918+516$ & $\mathrm{~N}, \mathrm{~S}$ & $180 \pm 5$ & $75 \pm 5$ & $210 \pm 10$ & $90 \pm 10$ & 7 & $2.00 \pm 0.11$ & $1.35 \pm 0.14$ & $37.1 \pm 2.9$ & $16.8 \pm 2.4$ \\
\hline B $2043+749$ & $\mathrm{~N}, \mathrm{~S}$ & $195 \pm 20$ & $115 \pm 15$ & $195 \pm 20$ & $150 \pm 15$ & 11 & $1.62 \pm 0.18$ & $1.80 \pm 0.17$ & $24.2 \pm 4.0$ & $30.2 \pm 4.0$ \\
\hline B $2147+816$ & $\mathrm{~N}, \mathrm{~S}$ & $370 \pm 20$ & $120 \pm 10$ & $310 \pm 20$ & $120 \pm 10$ & 9 & $0.94 \pm 0.08$ & $1.04 \pm 0.09$ & $8.1 \pm 0.9$ & $10.1 \pm 1.2$ \\
\hline
\end{tabular}

References: (1) 1.4-GHz WSRT data (Paper I); (2) Jägers 1986; (3) Vigotti et al. 1989; (4) Lara et al. (in preparation); (5) Mack et al. 1997; (6) FIRST survey; (7) 1.4-GHz WSRT data (unpublished); (8) Parma et al. 1996; (9) NVSS survey; (10) Röttgering et al. 1996; (11) Riley \& Warner 1990.

GRGs in our sample and we relate this to the properties of their environment, the IGM.

\subsection{Energy densities of the radio lobes}

We have calculated the equipartition energy densities and magnetic field strengths in the lobes of the FRII-type GRGs in our sample. We have used a method similar to that outlined by Miley (1980). To determine the luminosity of each lobe, we have used the integrated $325-\mathrm{MHz}$ flux densities (see Table 3). The spectral index of each lobe has been calculated between $325 \mathrm{MHz}$ and $10.5 \mathrm{GHz}$. In case of significant steepening of the radio spectrum at frequencies below $10.5 \mathrm{GHz}$ this value will be too low, but the influence of this on the result is only marginal (see Miley 1980).

The volumes of the radio lobes have been estimated assuming a cylindrical morphology. The width has been taken as the deconvolved average full width of the lobe, measured between the $3 \sigma$ contours on a radio contour map. The length of the lobe, whenever a clear gap exists between the core and the tail of the radio lobe (see, e.g., B $0648+733$, B 2147+816), has been taken as the distance between the innermost and outermost edge of the lobe at $325 \mathrm{MHz}$. The radio axes have been assumed to be in the plane of the sky; although this is certainly not the case for the majority of sources, the correction factors are not large compared to the uncertainties introduced by assuming cylindrical morphologies. These used lobe dimensions are quoted in Table 7. Further assumptions are a filling factor of the radiating particles of unity and an equal distribution of energy between electrons/positrons and heavy particles such as protons. The low and highfrequency cut-offs in the radio spectra have been set at $10 \mathrm{MHz}$ and $100 \mathrm{GHz}$, respectively.

In Table 7 we present the equipartition magnetic field strengths and energy densities of the lobes of the FRIItype sources in our sample. We find that the magnetic field strengths are low, typically a few $\mu \mathrm{G}$. Consequently, also the energy densities, which are directly derived from 

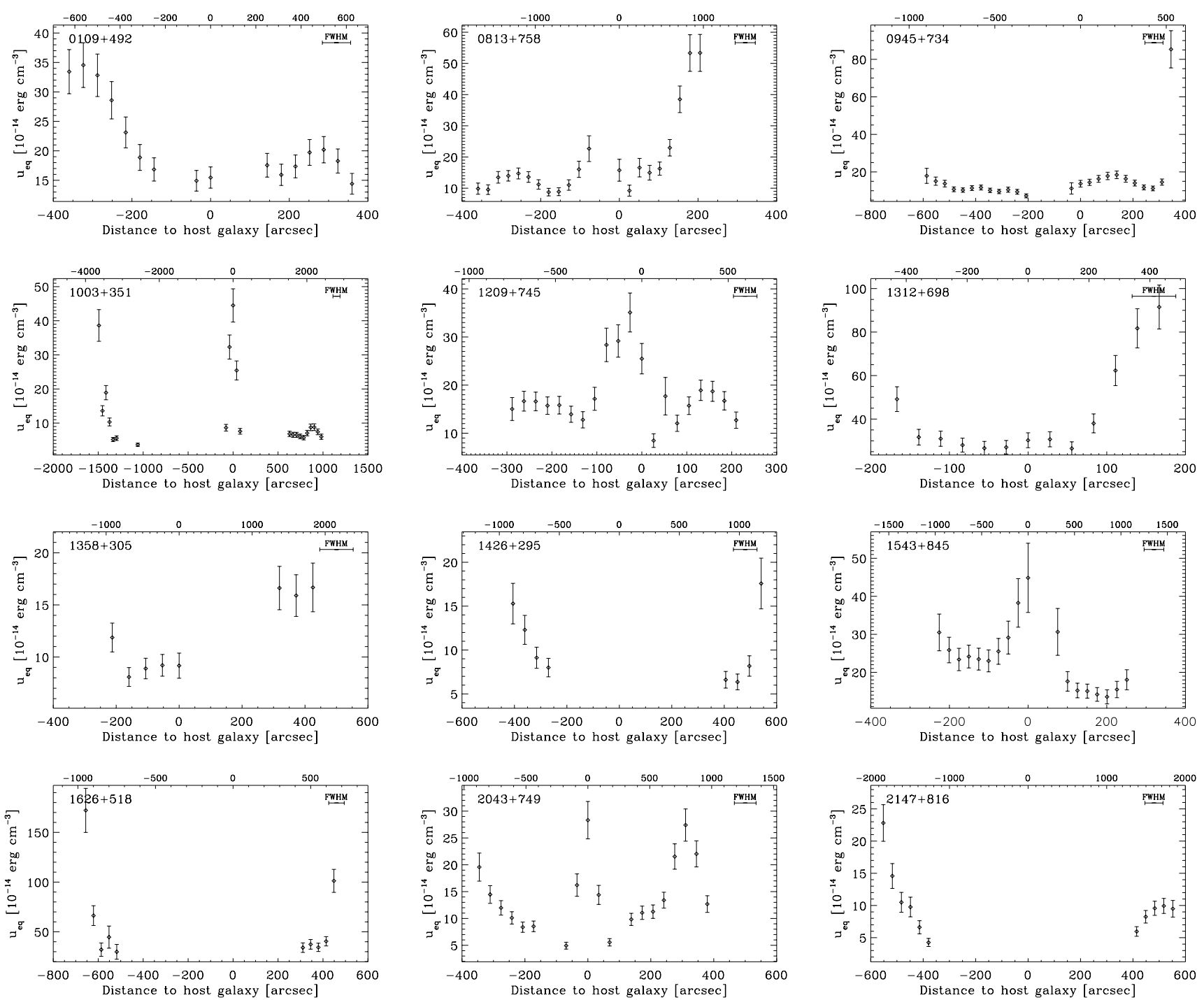

Fig. 9. Profiles of the equipartition energy density distribution along the major axes of several of the radio sources. The zeropoint is the position of the radio core. The numbers along the upper axis denote the distance from the core in units of kpc. Negative values are on that side of the source which is mentioned first in column two of Table 7

the magnetic field strengths $\left(u \propto B^{2}\right)$, are low. This is as expected since the GRGs are extremely large and not exceptionally powerful.

\subsection{Pressure gradients in the lobes}

The equipartition energy densities in Table 7 are the lobe volume averaged energy densities. Within the lobes, however, large variations may be present. To investigate this, we have made profiles of the equipartition energy density for the FRII-type sources above $7^{\prime}$ in size. We have used the same method as we have used to find the spectral index profiles in Sect. 5.1, i.e. by superposing on the source an array of boxes of width half the FWHM beamsize. In each box we have determined the average deconvolved width of the lobe by measuring the distance be- tween the $3 \sigma$ outer contours, and we have integrated the $325 / 354-\mathrm{MHz}$ flux density. The spectral index of each box was calculated between $325 / 354 \mathrm{MHz}$ and $10.5 \mathrm{GHz}$. In the case of B $0813+758$ and B $1209+745$ we used the 1.4-GHz NVSS map as the high-frequency map in order to employ the higher resolution available. In all cases the radio maps were first convolved to the lowest resolution map used. Only in boxes where the integrated flux density at both frequencies exceeded $3 \sigma$, the spectral index was calculated and the equipartition energy density determined. In case of the sources B $0050+402$ and B $0648+733$, the deconvolved lobe widths were below zero, and we have therefore omitted these sources from further analysis. The reason for this is not entirely clear to us.

The profiles of the equipartition energy density for the remaining sources are presented in Fig. 9. In some cases, 
such as B 0813+758, B 0945+734 and B 1312+698, we find the largest values of $u_{\text {eq }}$ at the heads of the lobes. In some other cases, such as B 1209+745 and B 2043+749, we clearly detect the influence of the radio core. When we compare the lobe averaged energy densities as presented in Table 7 with the average value of the profiles in the plots, we find that the differences, as expected, are relatively small. In only a few cases we find discrepancies, exceeding a factor of two, and these are mostly due to the difference in the assumed geometry of the lobes.

In a relativistic plasma, such as which constitutes the radio lobes, the pressure, $p$, is directly related to the energy density by $p=\frac{1}{3} u_{\text {eq }}$. Therefore, the plots in Fig. 9 also show the behaviour of the lobe pressure as a function of position along the radio axis. Since we have used a relatively low frequency for the flux density measurements and we have measured the spectral index for each plotted point separately, the results should not be highly sensitive to the increased effect of spectral ageing of the electrons towards the radio core.

If the host galaxy is at the center of a cluster, or if it has an extensive gaseous halo, hydrostatic pressure equilibrium requires a radial decrease of the pressure. The gradient should be strongest at small radii, around the core radius of the gas distribution. If the pressures in the bridges of the lobes are close to pressure equilibrium with their surrounding, it is expected that this gradient should be reflected in the lobe pressures at small radii.

We do not find such a behaviour in the majority of our sources. In the few cases where the energy density appears to increase near the position of the host galaxy this can be attributed to the presence of a radio core and/or radio jets (e.g. B $1209+745$ and B $2043+749$ ). Only in B $1543+845$ and in the eastern lobe of B $0813+758$ we observe a small increase in the pressure at small radii which cannot be related to a strong radio core or a jet (L. Lara, priv. comm.), and which might thus indicate the presence of a pressure gradient in the environment.

In most sources the energy density actually increases with increasing distance from the host galaxy. This indicates that the lobes must be overpressured with respect to their environment, even at small radii, and that there are pressure gradients in these lobes: The heads of the lobes have much higher pressures than the bridges.

\subsection{The density of the environment of the lobes}

From the ages, advance velocities and energy densities of the lobes we can estimate the density of the environment. We assume that the propagation of the head of the lobe is governed by a balance between the thrust of the jet and the ram-presssure exerted by the environment. In this case, the external density, $\rho_{\mathrm{a}}$, is given by $\rho_{\mathrm{a}}=\Pi_{\mathrm{j}} /\left(A_{\mathrm{h}} v_{\mathrm{h}}^{2}\right)$, where $\Pi_{\mathrm{j}}$ is the thrust of the jet, $A_{\mathrm{h}}$ is the area of the bowshock and $v_{\mathrm{h}}$ is the advance velocity of the head of the lobe. The thrust $\Pi_{\mathrm{j}}$ is given by $Q_{\text {jet }} / v_{\mathrm{j}}$, with $v_{\mathrm{j}}$ the velocity of the material in the jet, which we assume to be $c$, the velocity of light, and $Q_{\text {jet }}$, which is the amount of energy delivered by the jet per unit time, or the jet power. We can estimate the jet power by dividing the total energy contents of the lobes of the radio source by the age of the source. An important factor in this is the efficiency with which jet energy is converted to radiation. A conservative choice is given by Rawlings \& Saunders (1991): Half of the energy goes into the expansion of the lobe and the other half into radiation. Blundell et al. (1999), on the basis of radio source modelling, estimate values between 0.3 and 0.6 which agrees with a value of 0.5 . We therefore assume that the jet power is twice as high as the energy content of a radio lobe, divided by its age. The energy content can be estimated by multiplying the equipartition energy density with the volume of the lobe.

Since $\rho_{\mathrm{a}} \propto A_{\mathrm{h}}^{-1} \propto D_{\mathrm{h}}^{-2}$ (where $D_{\mathrm{h}}$ is the diameter of the impact area), $D_{\mathrm{h}}$ plays an important role in determining the ambient density. We cannot constrain it directly from our observations, but a good estimate is given by the size of the observable hotspot (e.g. Hardcastle et al. 1998). High-resolution observations of the hotspots in B 1312+698 can be found in Saunders et al. (1987). These show that the size of the hotspots is less than $15 \mathrm{kpc}$. Arcsecond resolution observations of the source B 2043+748 give similar constraints (Riley et al. 1988). Further, Hardcastle et al. (1998) find a positive correlation between the linear size of a source and the diameter of the hotspots in a sample of powerful 3CR FRII-type radio sources at $z<0.3$. This is confirmed for a larger sample of sources by Jeyakumar \& Saikia (2000). Based on these results, we have used a diameter of $5 \mathrm{kpc}$ to calculate the ambient densities of the lobes. For the age of a source, we have used the average age of both its lobes, and of both, CI and JP, ageing models. We have used the ages calculated with an internal magnetic field strength of $B_{\mathrm{MWB}} / \sqrt{3}$, since these are upper limits to the age and thus provide lower limits to $Q_{\text {jet }}$ and $v_{\mathrm{h}}$. Using this average age we have calculated the average jet power of the two lobes, which we assume to be equal on both sides of the source. The resulting densities are presented in Table 8 .

We find particle densities between $10^{-5}$ and $110^{-4} \mathrm{~cm}^{-3}$. They have been calculated using a mean atomic mass of $1.4 \mathrm{amu}$ in the environment of the radio sources. The densities are in good agreement with the results of Mack et al. (1998). If we assume a temperature in the IGM of a few $10^{6} \mathrm{~K}$ (e.g. Cen \& Ostriker 1999), the thermal pressure in the environment would be $\sim 210^{-14} \mathrm{dyn}_{\mathrm{cm}^{-2}}$ for a particle density of $410^{-5} \mathrm{~cm}^{-3}$. Such low pressures support the indications in Sect. 6.2 that the radio lobes must be overpressured with respect to the IGM. 
Table 8. Properties of the environment of the seven GRGs with useful lobe velocity and age determination. Column 1 gives the name of the source. Column 2 gives the volume, $V$, of the radio source. Column 3 gives the total energy, $u_{\text {tot }}$, of the radio lobes, defined as the equipartition energy density times the volume. Column 4 gives the average age, $t$, of the lobe. Column 5 gives the jet power, $Q_{\text {jet }}$. Column 6 gives the ambient density, $\rho_{\mathrm{a}}$, of the lobe and Col. 7 gives the particle density, $n_{\mathrm{a}}$, assuming a mean particle mass of $1.4 \mathrm{amu}$. The densities have been calculated using the mean of the jet powers of the lobes printed in Col. 5 and a diameter of the jet impact area of $5 \mathrm{kpc}$

\begin{tabular}{|c|c|c|c|c|c|c|c|}
\hline \multirow{2}{*}{\multicolumn{2}{|c|}{$\begin{array}{c}(1) \\
\text { Source }\end{array}$}} & & & & $(5)$ & & \\
\hline & & $\begin{array}{c}V \\
{\left[10^{72} \mathrm{~cm}^{3}\right]}\end{array}$ & $\begin{array}{c}u_{\text {tot }} \\
{\left[10^{59} \text { erg }\right]}\end{array}$ & $\begin{array}{c}t \\
{\left[10^{7} \mathrm{yr}\right]}\end{array}$ & $\begin{array}{c}Q_{\text {jet }} \\
{\left[10^{44} \text { erg s s}^{-1}\right]}\end{array}$ & {$\left[10^{-29} \stackrel{\rho_{\mathrm{a}}}{\left.\mathrm{gr} \mathrm{cm}^{-3}\right]}\right.$} & {$\left[10^{-5} \mathrm{~cm}_{\mathrm{a}}^{-3}\right]$} \\
\hline \multirow{2}{*}{ В $0109+492$} & $\mathrm{~N}$ & $1.73 \pm 0.06$ & $3.34 \pm 0.16$ & $9.3 \pm 0.6$ & $2.16 \pm 0.16$ & $10.7 \pm 1.1$ & $4.56 \pm 0.47$ \\
\hline & $\mathrm{S}$ & $1.73 \pm 0.06$ & $2.99 \pm 0.13$ & $9.3 \pm 0.6$ & $2.16 \pm 0.16$ & $10.7 \pm 1.1$ & $4.56 \pm 0.47$ \\
\hline \multirow[t]{2}{*}{ В $0813+758$} & $\mathrm{E}$ & $5.91 \pm 1.26$ & $16.5 \pm 4.5$ & $7.3 \pm 0.6$ & $10.2 \pm 2.7$ & $4.64 \pm 1.58$ & $1.98 \pm 0.67$ \\
\hline & $\mathrm{W}$ & $3.31 \pm 0.52$ & $7.48 \pm 1.54$ & $7.3 \pm 0.6$ & $10.2 \pm 2.7$ & $13.3 \pm 3.8$ & $5.68 \pm 1.62$ \\
\hline B $1003+351$ & $\mathrm{~W}$ & $37.8 \pm 3.4$ & $17.4 \pm 2.2$ & $9.8 \pm 0.3$ & $10.2 \pm 1.4$ & $2.88 \pm 0.40$ & $1.24 \pm 0.16$ \\
\hline \multirow[t]{2}{*}{ B $1209+745$} & $\mathrm{~N}$ & $1.76 \pm 0.38$ & $2.69 \pm 0.72$ & $9.9 \pm 0.9$ & $1.67 \pm 0.39$ & $5.74 \pm 1.52$ & $2.45 \pm 0.65$ \\
\hline & $\mathrm{S}$ & $1.48 \pm 0.18$ & $2.50 \pm 0.39$ & $9.9 \pm 0.9$ & $1.67 \pm 0.39$ & $16.9 \pm 4.1$ & $7.22 \pm 1.75$ \\
\hline \multirow[t]{2}{*}{ B $1312+698$} & $\mathrm{E}$ & $1.26 \pm 0.19$ & $2.75 \pm 0.51$ & $5.0 \pm 0.3$ & $3.83 \pm 0.77$ & $2.18 \pm 0.44$ & $9.31 \pm 0.89$ \\
\hline & $\mathrm{W}$ & $0.62 \pm 0.09$ & $3.11 \pm 0.57$ & $5.0 \pm 0.3$ & $3.83 \pm 0.77$ & $9.02 \pm 1.85$ & $3.85 \pm 0.79$ \\
\hline \multirow[t]{2}{*}{ B $1543+845$} & $\mathrm{~N}$ & $3.21 \pm 0.48$ & $12.3 \pm 2.5$ & $11.0 \pm 0.8$ & $9.18 \pm 2.83$ & $21.6 \pm 6.7$ & $9.22 \pm 2.84$ \\
\hline & $\mathrm{S}$ & $6.16 \pm 1.27$ & $19.5 \pm 5.9$ & $11.0 \pm 0.8$ & $9.18 \pm 2.83$ & $16.7 \pm 5.1$ & $7.13 \pm 2.20$ \\
\hline \multirow[t]{2}{*}{ B $2043+758$} & $\mathrm{~N}$ & $0.94 \pm 0.18$ & $2.27 \pm 0.58$ & $6.5 \pm 0.3$ & $3.48 \pm 0.82$ & $4.77 \pm 0.41$ & $2.04 \pm 0.18$ \\
\hline & $\mathrm{S}$ & $1.56 \pm 0.26$ & $4.74 \pm 1.01$ & $6.5 \pm 0.3$ & $3.48 \pm 0.82$ & $4.53 \pm 0.40$ & $1.93 \pm 0.16$ \\
\hline
\end{tabular}

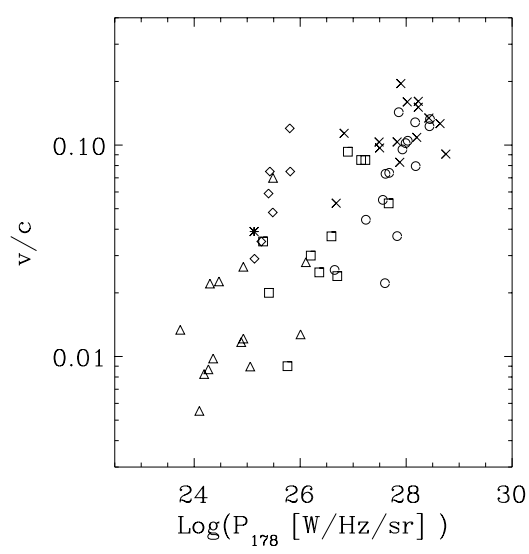

Fig. 10. The radio power against the lobe advance velocity for several samples of sources. Crosses: Liu et al. (1992); Circles: Leahy et al. (1989); Squares: Alexander \& Leahy (1987); Triangles: Parma et al. (1999); Diamonds: GRGs from this paper; Star: The GRG B 0313+683 from Schoenmakers et al. (1998)

\section{Discussion}

\subsection{The relation between lobe velocity and linear size}

In Sect. 5.2 we have derived the lobe advance velocities of seven of the GRGs in our sample. We have compared these velocities to those found in three samples of powerful FRII-type 3CR radio sources by Alexander \& Leahy (1987), Leahy et al. (1989) and Liu et al. (1992), and in a sample of lower luminosity sources presented by Parma et al. (1999). The sizes and radio powers of the sources in Leahy et al. and Parma et al. have been converted to a Hubble constant of $50 \mathrm{~km} \mathrm{~s}^{-1} \mathrm{Mpc}^{-1}$. Of the Parma et al. sample we have only used sources which are not of FRItype and which show a steepening of the radio spectrum from the hotspot to the core ("type 2" sources, in their notation). Also, the radio powers have been converted from 1.4 $\mathrm{GHz}$ to $178 \mathrm{MHz}$ using a spectral index of -0.8 . The sample of Alexander \& Leahy consists of radio sources with an angular size above $45^{\prime \prime}$, that of Leahy et al. of sources larger than $20^{\prime \prime}$ and that of Liu et al. of sources larger than $4^{\prime \prime}$. The sources 3C 154 and 3C 405 (Cyg A) are studied by both Alexander \& Leahy and Leahy et al.; we have used the better constrained values of the latter group. For all sources we have used the average age and velocities of the two lobes. For the GRGs we have taken the velocities derived using the JP ageing model, since this model has been used by Alexander \& Leahy, Leahy et al. and Liu et al. Lastly, we have added the advance velocity of the GRG B 0313+683 (Schoenmakers et al. 1998), which has been derived in a similar way as the GRGs presented here.

If we plot the radio power at $178 \mathrm{MHz}$ against the lobe advance velocity for all sources (Fig. 10) we find a strong correlation between these parameters. This correlation was noted before by Alexander \& Leahy (1987) and confirmed by Liu et al. (1992). We find that the GRGs agree with the correlation, but that they have the highest advance velocities for their radio powers. To find out 

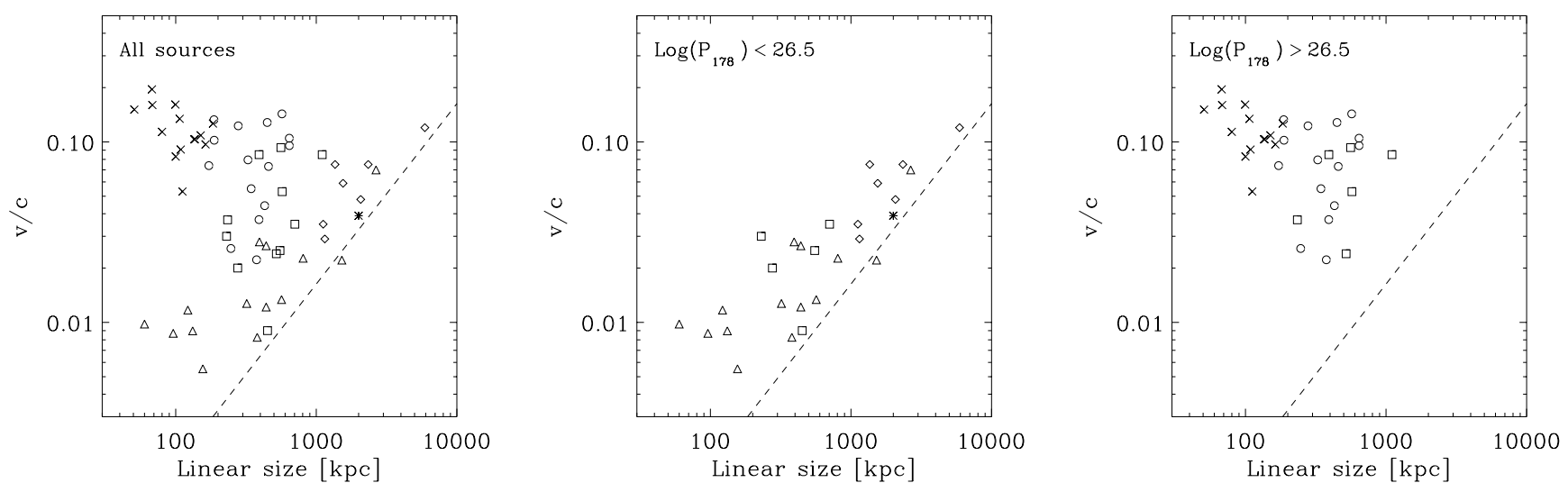

Fig. 11. The linear size of the radio sources against the lobe advance velocities. Symbols as in Fig. 10. The dashed line indicates the size a source can attain at an age of $10^{8}$ yr. a) (left) All sources. b) (middle) Sources with 178-MHz radio powers below $10^{26.5} \mathrm{~W} \mathrm{~Hz}^{-1}$ ster $^{-1}$, only. c) (right) Sources with $178-\mathrm{MHz}$ radio powers above $10^{26.5} \mathrm{~W} \mathrm{~Hz}^{-1}$ ster $^{-1}$

whether the advance velocity of the radio lobes is related to the linear size of a source, we have plotted these parameters for all sources in Fig. 11a. No correlation is found, although the spread in velocities appears to decrease with increasing linear size. However, when we separate the low luminosity from the high luminosity sources, we find a strong correlation for the low-luminosity sources (see Fig. 11b). We have set the separation at a radio power of $10^{26.5} \mathrm{~W} \mathrm{~Hz}^{-1} \mathrm{ster}^{-1}$, which is in between the highest radio power in the low-luminosity sample of Parma et al. (1999) and the lowest in the high-luminosity samples of Liu et al. (1992) and Leahy et al. (1989). For sources with higher radio powers the relation between velocity and source size seems to be inverted, although the correlation is much weaker (Fig. 11c).

At first sight, the correlation between linear size and lobe advance velocity for the low-luminosity sources suggests that radio sources increase their lobe advance velocity as they increase in size. However, the correlation can also be the result of two selection effect, provided that radio sources decrease in radio power as they increase in size and age (cf. Kaiser et al. 1997; Blundell et al. 1999). First, assuming that the radio power is proportional to the jet power and decreases with increasing linear size, large radio sources must have higher jet powers than small radio sources of similar observed radio power. Therefore, that fraction of large radio sources which are in a flux density limited sample must have the highest jet powers of all. If all radio sources are in similar environments, a higher jet power will most likely lead to a faster lobe advance; this yields the observed correlation. Second, since radio sources have a limited life-time, sources with low velocities will never become as large as sources with high lobe velocities. This is indicated by the dashed lines in Fig. 11, which represents an age of $10^{8} \mathrm{yr}$; not many sources are found to the right of this line. Of course, sources may also live longer than this, but they then have to drop below the sensitivity limit in order to disappear from the plot.

The above cannot explain the behaviour observed for the high power radio sources, though. There are several possible reasons for this, among which the following (see also Parma et al. 1999). First, the velocity estimates in these sources depend strongly on the assumed magnetic field strength in the lobes. This was taken as the equipartition field strength, but as shown by Liu et al. (1992), lowering this will drastically decrease the estimated velocities. Second, there may be strong backflows present in the lobes of the powerful sources (see also Scheuer 1995). This will also lead to an overestimation of the advance velocities since one actually measures the separation velocity between the head of the lobe and the material flowing back. Further, the mixing of old material with much younger, backflowing material near the radio core will lead to a further underestimation of the age of the source, and thus to an overestimation of the growth speed. Scheuer (1995) suggests that backflows are important in powerful sources such as present in the sample of Liu et al. (1992). It is unknown if strong backflows occur in less powerful sources as well. Lastly, in a flux density limited sample such as the 3CR, high-power sources are at higher redshifts than low power sources. Blundell et al. (1999) point out that this leads to the selection of younger and smaller radio sources with increasing redshift. Assuming that the radio power is directly related to the jet power, and that this is directly related to the advance velocities of the lobes, the lobes of powerful radio sources must have high velocities. The low-power sources discussed above are all at low redshift, so that this selection effect is not in operation.

\subsection{Spectral ages of radio sources}

Parma et al. (1999) find that the spectral ages of low power radio sources are well correlated with their linear sizes. 

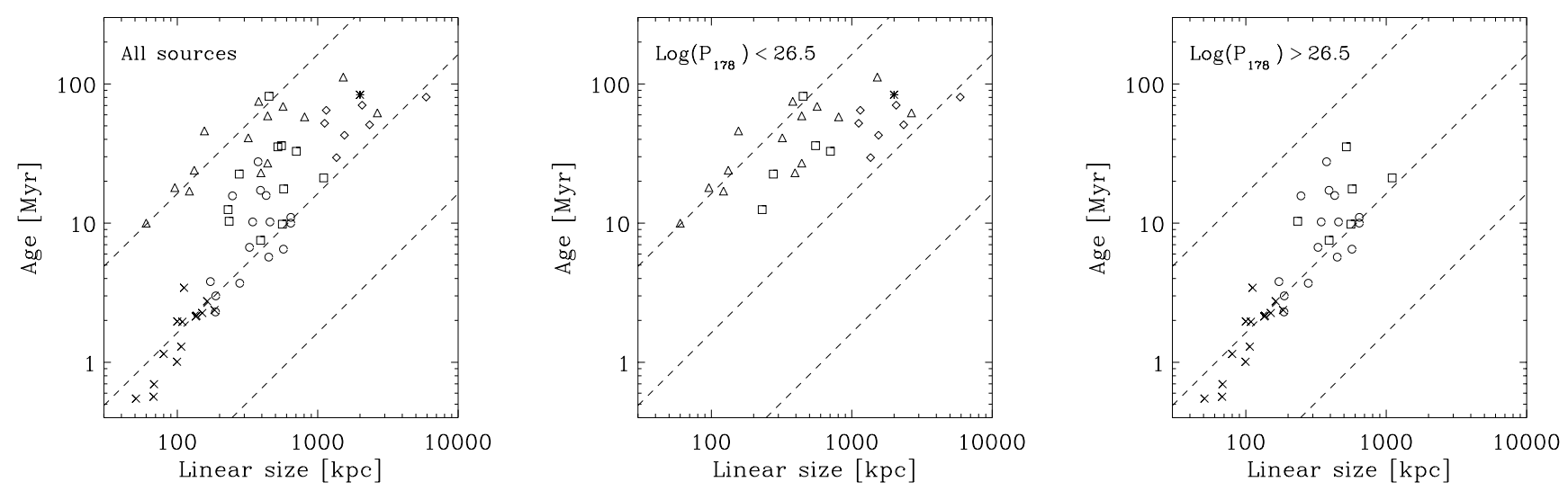

Fig. 12. The linear size of the radio sources against their spectral age. The symbols are as in Fig. 10. a) (left) All sources. b) (middle) Sources with $178-\mathrm{MHz}$ radio powers below $10^{26.5} \mathrm{~W} \mathrm{~Hz}^{-1}$ ster $^{-1}$, only. c) (right) Sources with $178-\mathrm{MHz}$ radio powers above $10^{26.5} \mathrm{~W} \mathrm{~Hz}^{-1} \mathrm{ster}^{-1}$. The diagonal lines indicate the relation for an expansion velocity of $0.01 c$ (upper line), $0.1 c$ (middle line) and $1 c$ (lower line)

In Fig. 12a we have plotted the linear sizes and spectral ages of the sources from the samples introduced in Sect. 7.1. We confirm the correlation between linear size and age, but we also find that the high-power sources have a stronger dependence on the linear size (see Figs. 12b and c). This is due to the higher lobe velocities, the cause of which we have discussed in Sect. 7.1. We find that the GRGs are among the oldest sources in this plot, although their ages are not extreme as compared to the sources from Parma et al. This is related to the correlation between linear size and lobe velocity (see Fig. 11b). Only large sources with high jet powers will end up in our GRG sample, which probably implies that their lobes advance faster (see previous section). This will introduce a bias in our sample towards sources which have higher advance velocities and thus lower ages. A multi-frequency study of GRGs at lower flux densities (such as those presented in Paper I) would be valuable to test the strength of this bias.

\subsection{Homogeneity of the Mpc-scale enviroment of GRGs}

In Sect. 4.1 we have presented the armlength and bending angle asymmetries of the GRGs and that of a sample of smaller-sized 3CR sources from Best et al. (1995). We find that the GRGs are slightly more asymmetric in their armlengths, although the bending angle distributions are similar. The radio lobes of GRGs have expanded well out of any extended emission-line gas regions, which may contribute significantly to the observed distribution of armlength-asymmetries in smaller sized radio sources (e.g. McCarthy et al. 1991). Therefore, it can be expected that the asymmetries are dominated by orientation effects. If, however, the asymmetries in GRGs are environmental, they provide information on the homogeneity, on Mpc- scales, of the intergalactic medium (IGM) surrounding their host galaxies.

We have attempted to fit the distribution of armlength asymmetries for the 3CR sources and the GRGs (see Fig. 3a) using orientation only (cf. Best et al. 1995). We find that the best fit for our subsample of $3 \mathrm{CR}$ sources is obtained for a maximum angle of $43^{\circ}$ between the plane of the sky and the line of sight. This assumes that the lobe velocities in a single source are equal and distributed as determined by Best et al. Since all sources in this 3CR subsample are radio galaxies, this agrees with the orientation dependent unification scheme (e.g. Barthel 1989). For the GRGs no satisfactory fit can be found. The main reason for this is the smaller number of sources in the first bin (see Fig. 3a), as compared to the second bin. To explain the observed distribution of the armlength asymmetries with equal advance velocities of the two lobes would require the introduction of a minimal allowed angle of $\sim 14^{\circ}$ between the radio axis and the plane of the sky. We therefore conclude that orientation effects alone cannot explain the observed distribution of armlengths in GRGs.

Best et al. (1995) show how the presence of nonlinearity in radio sources alters the distribution of armlength asymmetries. For the GRGS, however, the incorporation of the bending angles presented in Table 4 does not improve the fit significantly. As shown by Best et al., bending angles have the strongest effect on the observed armlength asymmetry distribution for sources oriented well away from the plane of the sky. For sources close to the plane of the sky, an increase of the maximum bending angle shifts the distribution of armlength asymmetries more towards $x=0$, which is opposed to what we observe.

These results suggest that the observed asymmetries must, at least partly, be due to asymmetries in the lobe advance velocities of the GRGs. This does not necessarily imply the existence of large-scale inhomogeneities in the 
IGM. Other possibilities are a difference in the efficiency of the energy transport down the jet, or of the jet opening angle. Both can influence the thrust produced by the jet material at the head of the lobe which influences the advance velocity.

In Sect. 4.1 we have investigated if the armlength asymmetry is related to the lobe flux density and spectral index asymmetries. Our most significant result is that the shorter arm tends to have the steepest radio spectrum. Can this be explained by environmental asymmetries? If there is a higher density on one side of the source, the radio lobe on that side may be slowed down and better confined than its counterpart on the other side. Provided that the magnetic field is frozen into the plasma, adiabatic expansion of a radio lobe results in a decrease of the magnetic and particle energy density (since $B \propto\left(r / r_{0}\right)^{-2}$ and $E \propto\left(r / r_{0}\right)^{-1}$, where $r_{0}$ is the initial radius and $r$ the radius after expansion, assuming spherical expansion; Scheuer \& Williams 1968). This implies that the particles responsible for the emission at a fixed frequency must have higher energies themselves (emitted frequency $\propto E^{2} B$ ), of which there are less available. These two mechanisms lead to a shift of the emitted spectrum to both lower intensities and lower frequencies. Since spectral ageing itself produces a convex spectral shape, the shift of the spectrum as a whole to lower frequencies leads to a steeper observed spectrum in case of an adiabatically expanded lobe as compared to a lobe which has not expanded, or by a lesser amount (e.g. Scheuer \& Williams 1968). This is contrary to what we find for the GRGs, arguing against a purely environmental origin for the shortest arm to have the steepest spectrum.

A second possibility is that maybe not the expansion of the lobe as a whole is important, but only the expansion of the radiating plasma as it leaves the overpressured hotspot area. In a higher density environment, and thus presumably higher pressure environment, the pressure in the hotspot will be larger as well (strong shock condition). If the pressure in the lobes is roughly equal in both lobes, then the drop in pressure of the plasma leaving the shock (or hotspot) will be larger on the side with the higher ambient density. Hence, the plasma will undergo a larger amount of expansion. This expansion will be relatively fast and will thus closely resemble the case of adiabatic expansion described above, i.e. the somewhat aged particle energy spectrum will be shifted towards lower energies. This will result in a steeper lobe spectrum on the side of the source with the shortest arm, as observed.

Since we have not yet enough information on the intrinsic properties of the jets and hotspots of the GRGs, we can not conclude that there are large scale inhomogeneities in their Mpc-scale environment. More detailed radio, and perhaps X-ray observations are necessary.

\subsection{Evolution of the environment of GRGs}

In an adiabatically expanding Universe filled with a hot, diffuse and uniform intergalactic medium, the pressure, $p_{\text {IGM }}$, should increase as a function of redshift, $z$, as $p_{\text {IGM }} \propto(1+z)^{5}$ (e.g. Subrahmanyan \& Saripalli 1993). In order to measure this effect using radio lobes, it is necessary to investigate which sources have the lowest radio lobe pressures. Radio source evolution models predict a decrease in the lobe pressure with increasing linear size (e.g. Kaiser \& Alexander 1997), so that it can be expected that the largest radio sources have the lowest lobe pressures.

Cotter (1998) uses a large sample of radio sources, among which are GRGs out to redshifts of $\sim 1$, and shows that indeed the energy densities in the radio lobes of GRGs are lower than those in smaller sized radio sources. Also, he shows that the lower limit for the lobe pressures evolves so strongly with redshift that it does not contradict a $(1+z)^{5}$ relation. This prompts the question if the equipartition pressures in the radio lobes of GRGs indeed indicate a strong evolution in their environments.

In Table 7 we have presented the energy densities in the lobes of the FRII-type GRGs in the 1-Jy sample. Although the range of redshift spanned by our sample is small $(0 \lesssim z \lesssim 0.3)$, a truly strong evolution of the energy density with redshift should show up. In Fig. 13a we have plotted for each source the average energy density of the two lobes against its redshift. We have used different symbols for sources with a linear size below and above $2 \mathrm{Mpc}$. The dotted line in the figure is the expected energy density in the lobes if the IGM has a presentday pressure, $p_{(\mathrm{IGM}, 0)}$, of $10^{-14}{\mathrm{dyn} \mathrm{cm}^{-2}}$ as suggested by Subrahmanyan \& Saripalli (1993), a redshift evolution as described above and if the lobes are in pressure equilibrium with the IGM. We find that the energy densities in the lobes of the sources with a size above $2 \mathrm{Mpc}$ are systematically lower than those in the lobes of the smaller sized sources, confirming the trend, already noted by Cotter (1998), for the largest radio sources. Also, the energy densities of the lobes of the GRGs indeed increase strongly with increasing redshift. We find that no source has a lobe energy density which would place it below the line predicted by a $(1+z)^{5}$ IGM pressure evolution with

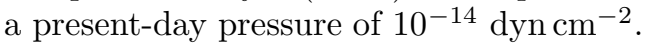

An important issue is how the selection effects affect the results. The equipartition energy density, $u_{\text {eq }}$, is related to the total power, $P$, and the volume, $V$, of a radio source by $u_{\text {eq }} \propto(P / V)^{4 / 7}$. The sources plotted are from a flux density limited sample $(S>1$ Jy at $325 \mathrm{MHz}$ ), which implies that sources at larger redshift have, on average, higher radio powers and thus higher energy densities at equal physical source dimensions.

In Fig. 13a we have plotted the expected equipartition energy density as a function of redshift (dashed line) for 

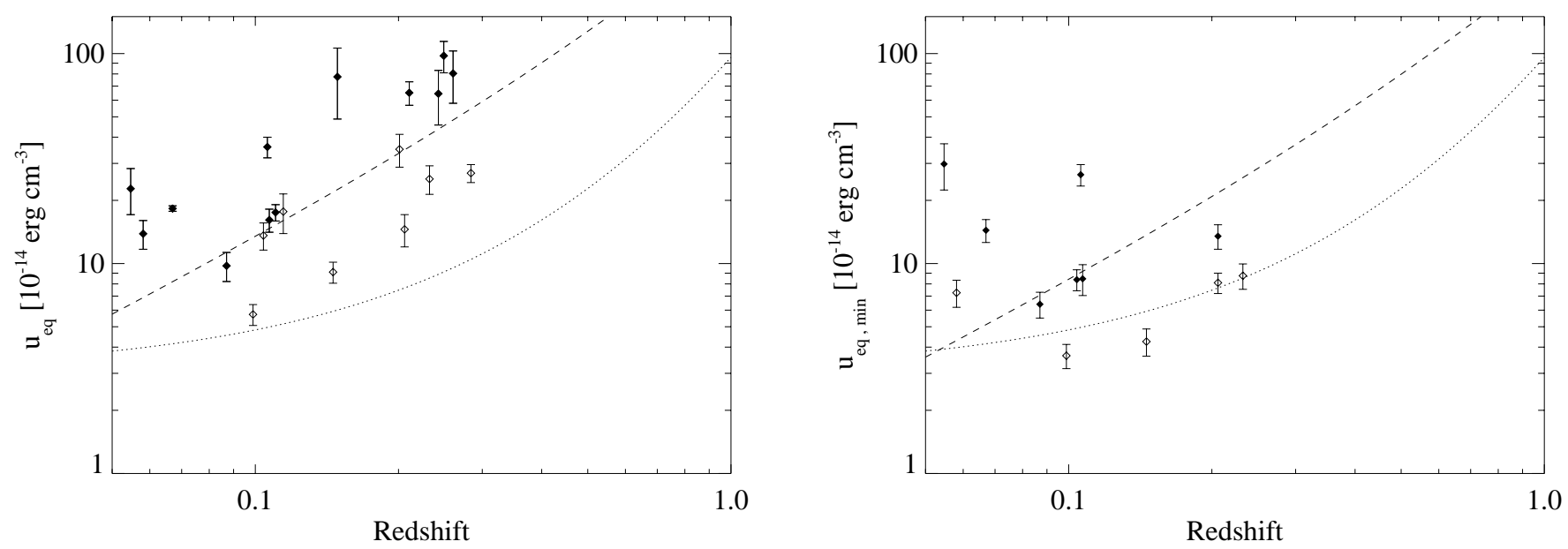

Fig. 13. Lobe-averaged equipartition energy densities of the FRII-type GRGs against redshift. a) (left) GRGs in the 1-Jy sample. Closed symbols are sources with a (projected) linear size between 1 and $2 \mathrm{Mpc}$, open symbols are sources larger than $2 \mathrm{Mpc}$. The dashed line indicates the expected behaviour for a source with a flux density, volume and spectral index as given by the median values of the sample. The dotted line indicates the lower limit if the pressure in the lobes is dominated by relativistic particles, the lobes are in pressure equilibrium with the IGM and the pressure, $p$, of the IGM follows the relation $p=1.010^{-14}(1+z)^{5}$ dyn $\mathrm{cm}^{-2}$. b) (right) The lowest measured energy densities in the lobes (see Fig. 9) against redshift. Symbols are as in (a)

a source with a flux density equal to the median flux density of the sample, a volume equal to the median volume and a spectral index equal to the median spectral index of the sources. Clearly, the slope and position of this line follows the redshift relation of the measured energy densities well. This suggests that the observed relation of the energy density with redshift is most likely a result from our use of a flux density and linear size limited sample, rather than of a cosmological evolution.

The energy densities are the intensity weighted averages over the two lobes. Since these values are sensitive to the contributions from the hotspots, the values in the fainter bridges of the lobes may be lower. From the profiles of the equipartition energy density presented in Fig. 9 we have taken the lowest found value for each source and plotted this in Fig. 13b. We find that most sources still lie well above the line describing the assumed $(1+z)^{5}$ pressure

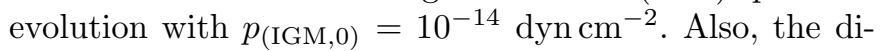
chotomy between sources smaller and larger than $2 \mathrm{Mpc}$ still exists, which indicates that even the lowest energy densities that we measure in the lobes are systematically lower in larger radio sources.

We can only conclude that there is no evidence in our sample for a cosmological evolution of the energy density in the lobes of GRGs, and that there is therefore also no evidence for a cosmological evolution of the pressure in the IGM. On the other hand, we are also aware that we cannot reject the hypothesis that the pressure of the IGM evolves as $p_{\text {IGM }} \propto(1+z)^{5}$, provided that the present-day value $p_{(\mathrm{IGM}, 0)} \lesssim 1.510^{-14} \mathrm{dyn} \mathrm{cm}^{-2}$.
The existence of a population of GRGs with lobe energy densities of $\lesssim 310^{-14} \mathrm{erg} \mathrm{cm}^{-3}$ at redshifts of at least 0.6 would challenge this hypothesis. Assuming a lengthto-width ratio of 3 , a size of $1.5 \mathrm{Mpc}$ and a spectral index of -0.8 , this requires that GRGs have to be found with a flux density of $\lesssim 200 \mathrm{mJy}$ at $325 \mathrm{MHz}$ at these redshifts, which is well above the sensitivity limit of WENSS and thus feasible. The flux density limit scales with the aspect ratio, $R$, as $R^{-3}$ and will thus rapidly drop below the survey limit of $\sim 25 \mathrm{mJy}$ (Rengelink et al. 1997) for more elongated sources. The only published sample of higher redshift GRGs is that of Cotter et al. (1996), which has been selected at $151 \mathrm{MHz}$ with flux densities between 0.4 and 1 Jy. These flux densities are higher than the above limit. The lobe pressures for the sources in this sample are presented by Cotter (1998). Indeed, he finds an apparent evolution of the lobe pressures in this sample, but this is therefore most likely also the result of the use of a flux density and linear size limited sample and not due to a true cosmological evolution of the IGM pressure.

Lastly, we have to mention the possibility that the environments of GRGs may be particularly underdense with respect to the environments of other radio sources. This has sometimes been suggested as the cause of the large size of the GRGs. If also the pressures in the ambient medium of GRGs indeed are typically lower, then the selection of radio sources as large as GRGs would be strongly biased towards regions with low pressures. Since this must be valid at all redshifts, we may be sampling the lowest pressure regions of the Universe only, which may be untypical of the general IGM. 


\section{Conclusions}

We have studied the radio properties of a complete sample of 26 giant radio galaxies. These have been selected from the WENSS survey at a flux density above $1 \mathrm{Jy}$ at $325 \mathrm{MHz}$ and an angular size above $5^{\prime}$. We have presented new radio observations of 18 of these sources at a frequency of $10.5 \mathrm{GHz}$, obtained with the 100-m Effelsberg telescope. These have been used in combination with available data from the WENSS, NVSS and GB6-surveys for a low-resolution (arcmin-scale) multi-frequency radio investigation of the properties of these sources. We have found the following:

1. The armlength asymmetries of GRGs are different from those of $3 \mathrm{CR}$ radio galaxies. The GRGs tend to be more asymmetric, which cannot be explained as an orientation effect only. We find that in 15 out of the 20 FRII-type GRGs in our sample, the radio lobe which has the steepest spectrum between $325 \mathrm{MHz}$ and $10.5 \mathrm{GHz}$ also has the shortest arm. We conclude that this cannot be result of a difference in the expansionrate of the two radio lobes, thereby excluding asymmetries in the environment as the major cause of this effect;

2. In profiles of the spectral index as a function of distance from the hotspots in the lobes, we find significant steepening of the spectrum away from the hotspot only in a few cases. Fitting these profiles with model spectra yields a typical advance velocities of $\sim 0.04 c$, and a spectral age of $80 \mathrm{Myr}$. Such large ages agree with what has been found for several other GRGs in the past;

3. We find a dichotomy between powerful $\left(P_{178}>\right.$ $10^{26.5} \mathrm{~W} \mathrm{~Hz}^{-1}$ ster $^{-1}$ ) and less powerful sources when we compare their lobe advance velocities, as deduced from spectral ageing studies, with their linear size. First, we find that for linear sizes around $100 \mathrm{kpc}$, the high power sources typically have much higher lobe velocities than the low power sources. This dichotomy disappears for larger sources. Further, less powerful sources show a strong correlation between source size and lobe advance velocity, which extends all the way to the largest sources, the GRGs. This may be largely due to the following: Slowly advancing sources may never grow out to Mpc sizes within the lifetime of the radio active phase of the $\mathrm{AGN}$;

4. Using the measured advance velocities, ages and energy contents of the lobes of the GRGs, we find a typical particle density in front of the lobes of a few times $10^{-5} \mathrm{~cm}^{-3}$. This is in agreement with earlier results on the density around the lobes of GRGs using similar methods (Mack et al. 1998). Assuming a temperature of a few times $10^{6} \mathrm{~K}$, the thermal pressure in a medium with a particle density of $410^{-5}$ would be $\sim 210^{-14}$ dyn $\mathrm{cm}^{-2}$. This lies below the typical lobe pressures we find from equipartition arguments.
Profiles of the equipartition energy density along the radio axis in the lobes indicate that there often is a strong pressure gradient in the lobes, with the hotspots having the highest pressures. Also this indicates that the radio lobes are overpressured with respect to their environment;

5. The lobe pressures show a strong correlation with redshift. This has been noted before by, e.g., Subrahmanyan \& Saripalli (1993) and Cotter (1998). We show that the correlation in our sample can be explained by two effects: The use of a flux density and linear size limited sample and the method by which the equipartition lobe pressures are calculated. We find therefore no evidence for a cosmological evolution of the IGM pressure between $z=0$ and $z=0.3$. Our observations agree with a present day value of the IGM pressure of $\sim 10^{-14}$ dyn $\mathrm{cm}^{-2}$.

This has been the first study of its kind employing a complete and relatively large sample of GRGs. The main result is that on basis of the data presented here we find that GRGs are both old sources, in terms of their spectral age, and that they are situated in a relatively low density environment, but also that neither of these two properties are extreme. Therefore, based on the study presented here, their large size probably results from a combination of these properties.

Acknowledgements. KHM was supported by the Deutsche Forschungsgemeinschaft, grant KL533/4-2 and by the European Commission, TMR Programme, Research Network Contract ERBFMRXCT96-0034 "CERES". The Westerbork Synthesis Radio Telescope (WSRT) is operated by the Netherlands Foundation for Research in Astronomy (NFRA) with financial support of the Netherlands Organization for Scientific Research (NWO). The National Radio Astronomy Observatory (NRAO) is operated by Associated Universities, Inc., and is a facility of the National Science Foundation (NSF). This research has made use of the NASA/IPAC Extragalactic Database (NED) which is operated by the Jet Propulsion Laboratory, California Institute of Technology, under contract with the National Aeronautics and Space Administration. We acknowledge the use of NASA's SkyView facility (http://skyview.gsfc.nasa.gov) located at NASA Goddard Space Flight Center.

\section{References}

Alexander P., Leahy J.P., 1987, MNRAS 225, 1

Baars J.W.M., Genzel R., Pauliny-Toth I.I.K., Witzel A., 1977, A\&A 61,99

Barthel P.D., 1989, ApJ 336, 606

Barthel P.D., Miley G.K., Jägers W.J., Schilizzi R.T., Strom R.G., 1985, A\&A 148, 243

Best P.N., Bailer D.M., Longair M.S., Riley J.M., 1995, MNRAS 275, 1171

van Breugel W.J.M., Willis A.G., 1981, A\&A 96, 332

Blundell K., Rawlings S., Willott C.J., 1999, AJ 117, 677

de Bruyn A.G., 1989, A\&A 226, L13 
Cen R., Ostriker J.P., 1999, ApJ 514, 1

Condon J.J., Cotton W.D., Greisen E.W., Yin Q.F., Perley R.A., Taylor G.B., Broderick J.J., 1998, AJ 115, 1693

Cotter G., 1998, in: Observational cosmology with the new radio surveys, Bremer M.N., Jackson N., Pérez-Fournon I. (eds.). Kluwer, p. 233

Cotter G., Rawlings S., Saunders R., 1996, MNRAS 281, 1081

Emerson D.T., Klein U., Haslam C.G.T., 1979, A\&A 76, 92

Fanaroff B.L., Riley J.M., 1974, MNRAS 167, 31

Gregorini L., Klein U., Parma P., Wielebinski R., Schlickeiser R., 1992, A\&AS 94, 13

Gregorini L., Vigotti M., Mack K.-H., Zönnchen J., Klein U., 1998, A\&AS 133, 129

Gregory P.C., Scott W.K., Douglas K., Condon J.J., 1996, ApJS 103, 427

Hardcastle M.J., Alexander P., Pooley G.G., Riley J.M., 1998, MNRAS 296, 445

Hine R.G., 1979, MNRAS 189, 527

Ishwara-Chandra C.H., Saikia D.J., 1999, MNRAS 309, 1001

Jägers W.H., 1986, Ph.D. Thesis, University of Leiden

Jeyakumar S., Saikia D.J., 2000, MNRAS 311, 397

Kaiser C.R., Alexander P., 1997, MNRAS 286, 215

Kaiser C.R., Alexander P., 1999, MNRAS 305, 707

Kaiser C.R., Dennett-Thorpe J., Alexander P., 1997, MNRAS 292, 723

Klein U., Mack K.-H., 1995, in: Proceedings Workshop on "Multi-Feed Systems for Radio Telescopes", Tucson, Emerson D.T. (ed.), ASP Conf. Ser.

Klein U., Mack K.-H., Strom R., Wielebinski R., Achatz U., 1994, A\&A 283, 729

van der Laan H., Perola G.C., 1969, A\&A 3, 468

Lacy M., Rawlings S., Saunders R., Warner P.J., 1993, MNRAS 264,721

Laing R.A., Riley J.M., Longair M.S., 1983, MNRAS 204, 151

Leahy J.P., Muxlow T.W.B., Stephens P.W., 1989, MNRAS 239, 401

Liu R., Pooley G., Riley J.M., 1992, MNRAS 257, 545

Mack K.-H., Gregorini L., Parma P., Klein U., 1994, A\&AS 103,157

Mack K.-H., Klein U., O’Dea C.P., Willis A.G., 1997, A\&AS 123,423

Mack K.-H., Klein U., O'Dea C.P., Willis A.G., Saripalli L., 1998, A\&A 329, 431

McCarthy P.J., van Breugel W., Kapahi V.K., 1991, ApJ 371, 478

Miley G.K., 1980, ARA\&A 18, 165

Parma P., de Ruiter H.R., Mack K.-H., van Breugel W., Dey A., Fanti R., Klein U., 1996, A\&A 311, 49

Parma P., Murgia M., Morganti R., Capetti A., de Ruiter H.R., Fanti R., 1999, A\&A 344, 7

Perley R.A., Bridle A.H., Willis A.G., 1984, ApJS 54, 291

Rawlings S., Saunders R., 1991, Nat 349, 138

Rengelink R., Tang Y., de Bruyn A.G., Miley G.K., Bremer M.N., Röttgering H.J.A., Bremer M.A.R., 1997, A\&AS 124,259

Riley J.M., Warner P.J., Rawlings S., Saunders R., Pooley G.G., Eales S.A., 1988, MNRAS 236, 13p

Saripalli L., Mack K.-H., Klein U., Strom R., Singal A.K., 1996, A\&A 306, 708

Saripalli L., Subrahmanyan R., Hunstead R.W., 1994, MNRAS 269,37
Saunders R., Baldwin J.E., Warner P.J., 1987, MNRAS 225, 713

Scheuer P.A.G., 1995, MNRAS 277, 331

Scheuer P.A.G., Williams P.J.S., 1968, ARAA 6, 321

Schoenmakers A.P., 1999, Ph.D. Thesis, University of Utrecht

Schoenmakers A.P., Mack K.-H., Lara L., Röttgering H.J.A., de Bruyn A.G., van der Laan H., Giovannini G., 1998, A\&A 336,455

Schoenmakers A.P., de Bruyn A.G., Röttgering H.J.A., van der Laan H., 2000a (in preparation) (Paper I)

Schoenmakers A.P., de Bruyn A.G., Röttgering H.J.A., van der Laan H., Kaiser C.R., 2000b, MNRAS 315, 371

Strom R.G., Willis A.G., 1980, A\&A 85, 36

Subrahmanyan R., Saripalli L., 1993, MNRAS 260, 908

Subrahmanyan R., Saripalli L., Hunstead R.W., 1996, MNRAS 279,257

Willis A.G., Strom R.G., Bridle A.H., Fomalont E.B., 1981, A\&A 95, 250

\section{Appendix A: Radio maps of the 10.5-GHz Effelsberg observations}

Here we present the radio maps resulting from the $10.5 \mathrm{GHz}$ Effelsberg observations of 18 sources in our 1-Jy sample of Giant Radio sources (Figs. B1-B4). For all sources, two maps have been presented. The first, which is indicated by an "I" and has the name of the source on the plot as well, shows contours of total intensity, with $E$-field polarization vectors overplotted. The length of the vectors is proportional to the polarized intensity. The scale used is $1^{\prime \prime}=0.1 \mathrm{mJy}^{\text {beam }}{ }^{-1}$, except in the case of B $0157+405$ where $1^{\prime \prime}=0.05$ mJybeam $^{-1}$. The second plot, indicated by "P", shows contours of polarized intensity with $E$-field polarization vectors overplotted. Here, the length of the polarization vectors is proportional to the fractional polarization. The scale used is $1^{\prime \prime}=1 \%$ fractional polarization, except in the source B $0309+411$, where $1^{\prime \prime}=0.1 \%$ fractional polarization. All contours are drawn at intervals of a factor of $\sqrt{2}$, with the value of the lowest contour given in Table A1. In the case of B $0309+411$, contours above 16 times the lowest contour are drawn at intervals of a factor of 2. Dashed contours indicate negative flux densities; only the first negative contour has been drawn. References to similar $10.5-\mathrm{GHz}$ Effelsberg maps of the remaining eight sources in the 1-Jy sample are presented in the table.

\section{Appendix B: Notes on individual sources}

B 0050+402: A total intensity map has already been presented by Gregorini et al. (1998); here, we have added the polarization data. Both lobes are only weakly polarized.

B 0109+402: This source is also known as 3C 35 and is a fat double radio source (e.g. Jägers 1986). We have detected the core. The complicated polarization structure in the northern lobe results from a rapid 
Table A1. Flux density values of the lowest contours in the contour plots of the Effelsberg $10.5 \mathrm{GHz}$ data (Figs. B1-B4). Column 3 gives the flux of the lowest contour used in the total intensity $(I)$ plot in mJy beam ${ }^{-1}$; Col. 4 gives the flux density of the lowest contour in the polarized intensity $(P)$ contour plot in $\mathrm{mJy}_{\text {beam }}{ }^{-1}$; Col. 5 gives the reference to similar maps which have been presented elsewhere

\begin{tabular}{llccl}
\hline \hline IAU name & Alt. name & $(3)$ & $(4)$ & $(5)$ \\
\hline B 0050+402 & & 2.7 & 1.70 & Ref. \\
B 0055+300 & NGC 315 & & & Mack et al. 1997 \\
B 0109+492 & 3C 35 & 4.5 & 0.95 & \\
B 0136+396 & 4C 39.04 & & & Mack et al. 1994 \\
B 0157+405 & 4C 40.09 & 3.2 & 0.95 & \\
B 0211+326 & & 2.7 & 0.80 & \\
B 0309+411 & & 3.3 & 0.90 & \\
B 0648+733 & & 3.0 & 1.00 & \\
B 0658+490 & & 3.3 & 0.85 & \\
B 0745+560 & DA 240 & & & Mack et al. 1997 \\
B 0813+758 & & 3.0 & 0.90 & \\
B 0945+734 & 4 C 73.08 & & & Klein et al. 1994 \\
B 1003+351 & 3C 236 & & & Mack et al. 1997 \\
B 1209+745 & 4CT 74.17 & 3.0 & 0.90 & \\
B 1213+422 & & 3.9 & 1.25 & \\
B 1309+412 & & 3.9 & 1.55 & \\
B 1312+698 & DA 340 & 2.7 & 0.90 & \\
B 1358+305 & & & & Saripalli et al. 1996 \\
B 1426+295 & & 3.3 & 0.90 & \\
B 1450+333 & & 1.8 & 0.90 & \\
B 1543+845 & & 1.8 & 0.85 & \\
B 1626+518 & & 3.9 & 0.85 & \\
B 1637+826 & NGC 6251 & & & Mack et al. 1997 \\
B 1918+516 & & 3.9 & 1.20 & \\
B 2043+749 & 4 C 74.26 & & & Saripalli et al. 1996 \\
B 2147+816 & & 2.9 & 0.85 & \\
\hline \hline
\end{tabular}

change of the polarization angle with position, causing beam-depolarization (see the higher resolution maps of Jägers 1986).

B 0157+405: This source has been barely detected in our observations. We find a spectral index of $-0.99 \pm 0.04$ between $325 \mathrm{MHz}$ and $10.5 \mathrm{GHz}$, which is indeed rather steep.

$B 0211+326$ : This source is well detected in both total power and linearly polarized emission; the direction of the observed $E$-field is perpendicular to the radio axis in both lobes, as is usually found in the lobes of FRIItype sources.

$B 0309+411$ : This is a strongly core dominated giant radio source (de Bruyn 1989). Indeed, our radio map is dominated by the core emission as well. The faint extension towards the north-east is an artefact due to the beam.

$B 0648+733$ : This is one of our largest sources. The angle of the observed $E$-field is perpendicular to the radio axis in the south-west lobe, and parallel in the northeast lobe. Considering the high frequency of these observations, it is unlikely that this is caused by Faraday rotation and this configuration therefore must be internal.
B 0658+490: This source has a strange morphology (see Paper I). Here, only the compact central components are well detected. The core is unpolarized at our sensitivity, the other two components have an observed $E$-field parallel (middle component) and perpendicular (western component) to the radio axis.

$B 0813+758$ : This source displays a rather dominant radio core at $10.5 \mathrm{GHz}$. Of the two lobes, only the western one is detected in linearly polarized emission.

B 1209+745: The luminous central core/jet structure (see van Breugel \& Willis 1981) is unresolved. Polarized emission has been detected at the position of the jet and towards the southern lobe.

$B 1213+422$ : The radio core of this source is quite bright at $10.5 \mathrm{GHz}$. No significantly polarized features are detected.

B 1309+412: The total intensity map of this source has already been presented by Gregorini et al. (1998); here, we display the polarization data. Linear polarization has been detected in both lobes, with observed $E$-fields parallel to the radio axis.

$B 1312+698$ : This source is well detected in polarized emission, with an observed $E$-field direction perpendicular to the radio axis. The unresolved polarized feature in between the two lobes may be a signature of a jet.

$B 1426+295$ : The total intensity map shows the two radio lobes and the core of this large source. The $E$-field direction of the polarized emission in the two lobes is perpendicular to the radio axis.

$B 1450+333$ : This is one of the "double-double" radio sources presented in Schoenmakers et al. (2000b). The resolution of these observations is too low to investigate the properties of the inner structure in detail. However, we find polarized emission towards the inner structure. Polarized emission has also been detected towards both outer radio lobes, with $E$-field directions perpendicular to the radio axis.

B 1543+845: In total intensity this source looks like a normal FRII-type radio source. In polarized emission, only the northern lobe of this source is detected, with the $E$-field directed perpendicular to the radio axis.

$B 1626+518$ : The contour map shows the two lobes and the relatively bright radio core of this source. Also polarized emission has been detected in both lobes.

B 1918+516: Both lobes and a radio core are detected, although the latter is somewhat confused with a nearby source. Polarized emission has been detected towards both radio lobes, with $E$-field directions almost perpendicular to the radio axis.

$B 2147+816$ : The radio core and the two extended lobes of this $3.7 \mathrm{Mpc}$ large source are well detected. Polarized emission is detected towards both radio lobes, with $E$ field directions almost perpendicular to the radio axis. The southern lobe shows signs of a change in the orientation of the polarization angle at its southern edge. 

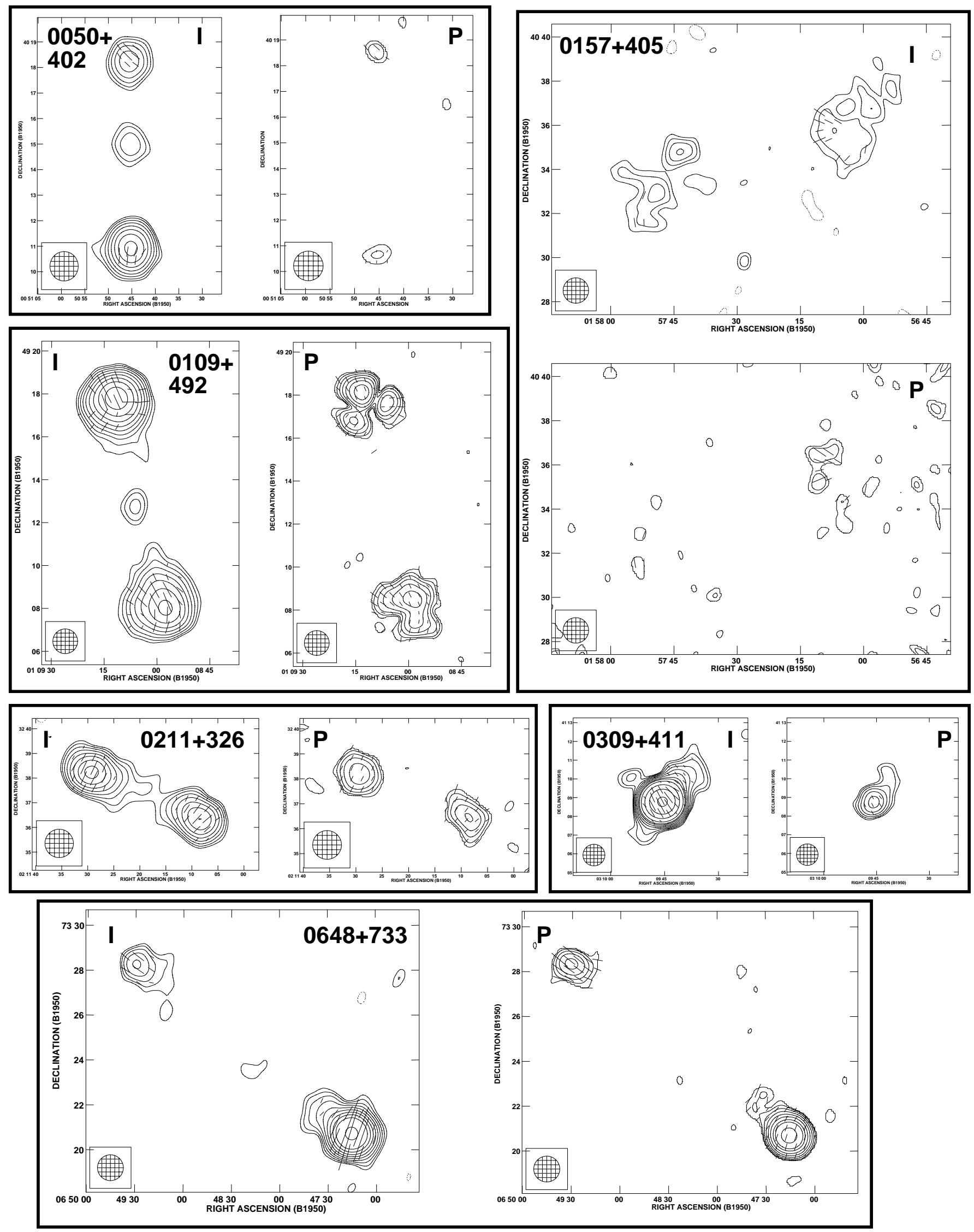

Fig. B1. Radio contourplots from the 10.5-GHz Effelsberg observations of the sources B $0050+402$, B 0109+492, B 0157+405, B $0211+326$, B 0309+411 and B 0648+733 

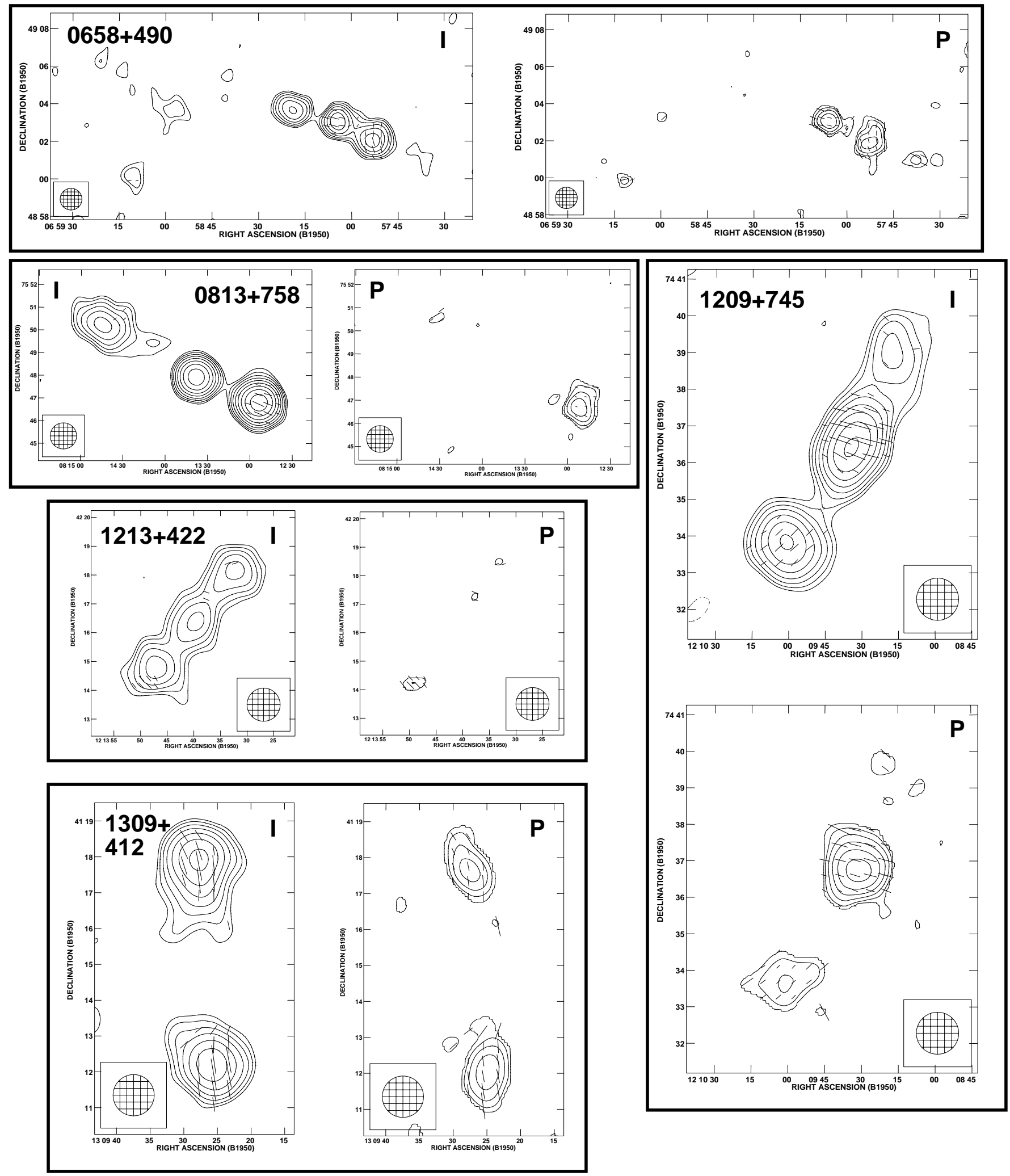

Fig. B2. Radio contourplots from the 10.5-GHz Effelsberg observations of the sources B 0658+492, B 0813+758, B 1209+745, B $1213+422$ and B $1309+412$ 

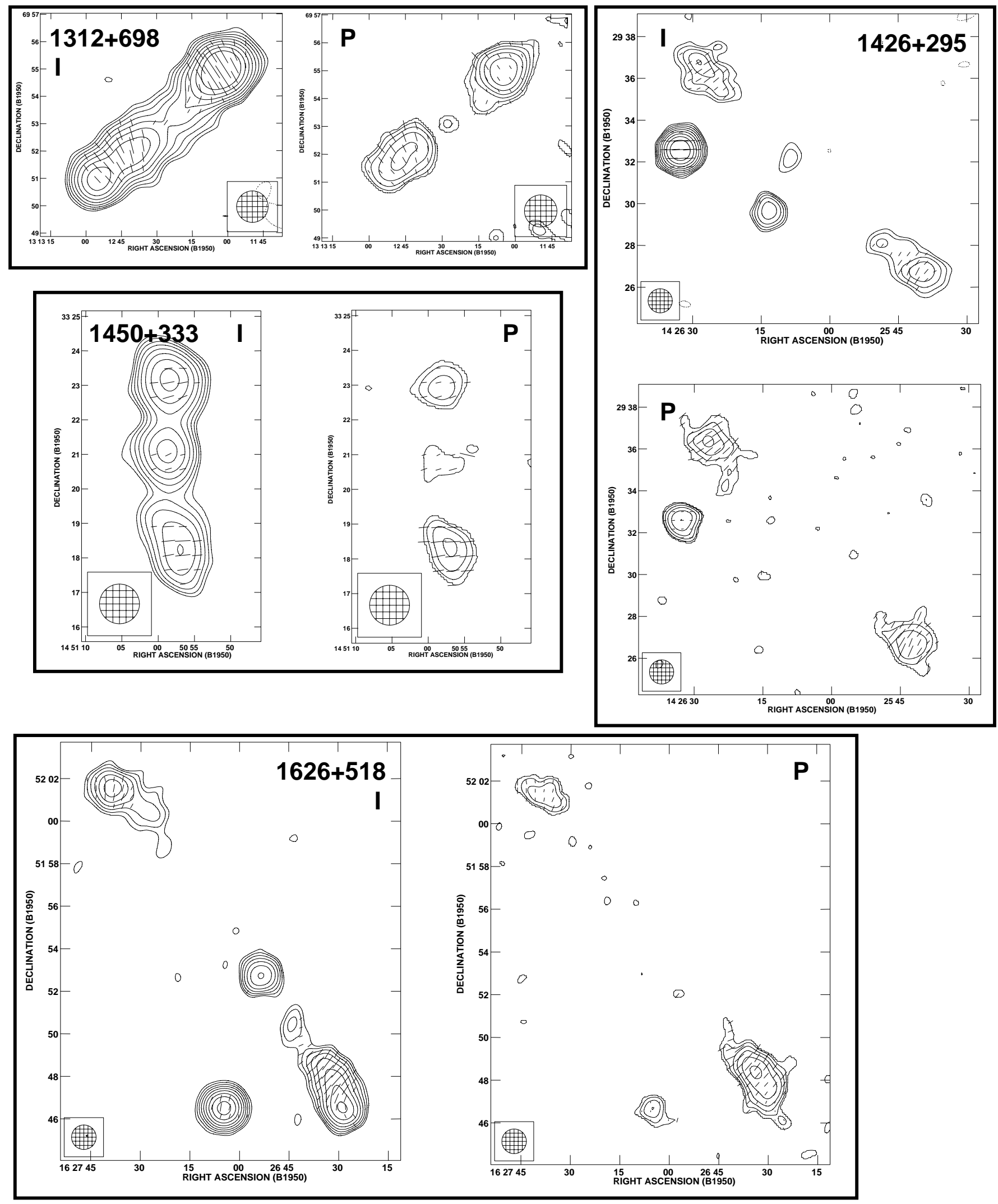

Fig. B3. Radio contourplots from the 10.5-GHz Effelsberg observations of the sources B 1312+698, B 1426+295, B $1450+333$ and B $1626+518$ 

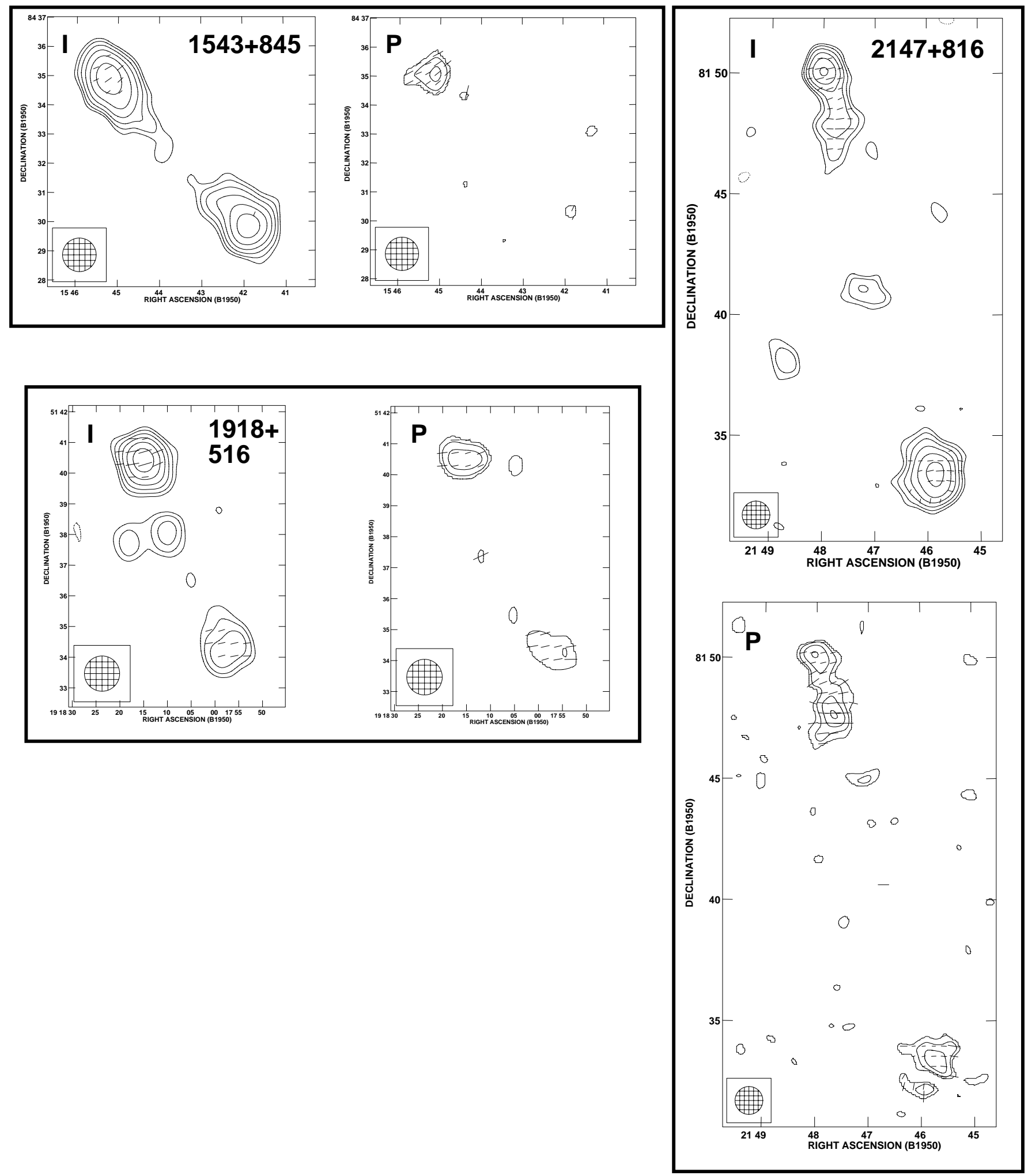

Fig. B4. Radio contourplots from the 10.5-GHz Effelsberg observations of the sources B 1543+845, B $1918+516$ and B $2147+816$ 